ARTICLE

\title{
Oxylipin metabolism is controlled by mitochondrial $\beta$-oxidation during bacterial inflammation
}

Mariya Misheva1, Konstantinos Kotzamanis (10 1,7, Luke C. Davies ${ }^{1,7}$, Victoria J. Tyrrell', Patricia R. S. Rodrigues (10 1, Gloria A. Benavides², Christine Hinz', Robert C. Murphy ${ }^{3}$, Paul Kennedy ${ }^{4}$, Philip R. Taylor (10) 1,5, Marcela Rosas ${ }^{1}$, Simon A. Jones ${ }^{1}$, James E. McLaren (1) ${ }^{1}$, Sumukh Deshpande ${ }^{1}$, Robert Andrews ${ }^{1}$, Nils Helge Schebb ${ }^{6}$, Magdalena A. Czubala ${ }^{1}$, Mark Gurney (D) ${ }^{1}$, Maceler Aldrovandi ${ }^{1}$, Sven W. Meckelmann ${ }^{1}$, Peter Ghazal (1) ${ }^{1}$, Victor Darley-Usmar ${ }^{2}$, Daniel A. White ${ }^{1 凶} \&$ Valerie B. O'Donnell (id ${ }^{1 \times}$

Oxylipins are potent biological mediators requiring strict control, but how they are removed en masse during infection and inflammation is unknown. Here we show that lipopolysaccharide (LPS) dynamically enhances oxylipin removal via mitochondrial $\beta$-oxidation. Specifically, genetic or pharmacological targeting of carnitine palmitoyl transferase 1 (CPT1), a mitochondrial importer of fatty acids, reveal that many oxylipins are removed by this protein during inflammation in vitro and in vivo. Using stable isotope-tracing lipidomics, we find secretion-reuptake recycling for 12 -HETE and its intermediate metabolites. Meanwhile, oxylipin $\beta$-oxidation is uncoupled from oxidative phosphorylation, thus not contributing to energy generation. Testing for genetic control checkpoints, transcriptional interrogation of human neonatal sepsis finds upregulation of many genes involved in mitochondrial removal of long-chain fatty acyls, such as ACSL1,3,4, ACADVL, CPT1B, CPT2 and HADHB. Also, ACSL1/ Acs/7 upregulation is consistently observed following the treatment of human/murine macrophages with LPS and IFN- $\gamma$. Last, dampening oxylipin levels by $\beta$-oxidation is suggested to impact on their regulation of leukocyte functions. In summary, we propose mitochondrial $\beta$ oxidation as a regulatory metabolic checkpoint for oxylipins during inflammation.

\footnotetext{
${ }^{1}$ Systems Immunity Research Institute and Division of Infection and Immunity, and School of Medicine, Cardiff University, CF14 4XN Cardiff, UK. ${ }^{2}$ Department of Pathology, University of Alabama at Birmingham, Birmingham, AL 35294, USA. ${ }^{3}$ Department of Pharmacology, University of Colorado Denver, Aurora, CO 80045 , USA. ${ }^{4}$ Cayman Chemical, 1180 E Ellsworth Rd, Ann Arbor, MI 48108, USA. ${ }^{5}$ UK Dementia Research Institute at Cardiff, Cardiff University, CF14 4XN Cardiff, UK. ${ }^{6}$ Chair of Food Chemistry, Faculty of Mathematics and Natural Sciences, University of Wuppertal, Gausstraße 20, 42119 Wuppertal, Germany. ${ }^{7}$ These authors contributed equally: Konstantinos Kotzamanis, Luke C. Davies.凶email: WhiteD13@cardiff.ac.uk; o-donnellvb@cardiff.ac.uk
} 
xygenated polyunsaturated fatty acids (PUFA) (oxylipins) are essential bioactive lipid mediators generated during inflammation/infection. They are generated by cyclooxygenases (COX), lipoxygenases (LOX), or cytochrome P450s (CYP), expressed in a variety of cells and tissues ${ }^{1-10}$. They signal through activation of $G$ protein-coupled receptors (GPCRs) at sub nM concentrations ${ }^{1,5-16}$. Oxylipin signaling requires deactivation; however, our understanding of how this happens during infection is poor. Systemic pathways for individual oxylipins, including thromboxane, prostacyclin and hydroxyeicosatetraenoic acids (HETEs) were uncovered in healthy humans decades ago ${ }^{17-20}$. There, infusion of exogenous labeled lipids enabled determination of half-life and metabolites, some of which appear immediately, slowly disappearing over several minutes ${ }^{21}$. Urinary metabolite analysis became the gold standard for whole body oxylipin analysis. Separately, peroxisomal $\beta$ oxidation of individual oxylipins was explored using liver microsomes. There, partial $\beta$-oxidation revealed truncated products termed dinors (minus 2 carbons) and tetranors (minus 4 carbons). These were identified as stable intermediates in tissue, plasma and urine ${ }^{21}$.

Aside from peroxisomes, mitochondria contain fully competent $\beta$-oxidation machinery, used for the first steps of FA-dependent energy metabolism, FA oxidation $(\mathrm{FAO})^{22,23}$. Here, FA are converted to acetyl-CoA which enters the tricarboxylic cycle (TCA) providing substrates for oxidative phosphorylation (OxPhos) ${ }^{24}$. Although mediated by distinct enzymes to peroxisomes, mitochondrial $\beta$-oxidation also involves sequential removal of 2 -carbon fragments from the carboxyl terminus. More recently, there has been significant interest in how mitochondrial FAO supports innate and adaptive immunity in $\mathrm{T}$ cells and macrophages ${ }^{25,26}$. Saturated FA such as palmitate or stearate are considered the main $\beta$-oxidation substrates; however, oxylipins generated abundantly during inflammation have not been considered ${ }^{19}$. We recently showed that endogenously generated platelet oxylipins are removed by carnitine palmitoyltransferase-1 (CPT1), the mitochondrial import protein for $\mathrm{FAs}^{27}$. However, it is not known whether mitochondria also remove oxylipins in other leukocytes, nor how this might be regulated during inflammation/infection, if this impacts lipid signaling, and if it supplies acetyl-CoA to OxPhos. These are important questions since large amounts of diverse oxylipins are generated during inflammation/infection, with downstream autocrine and paracrine signaling being a major contributor to the overall inflammatory response. Currently, there is significant interest in how mitochondria contribute to inflammation, and their role in immunometabolism $26,28,29$. One view is that in lipopolysaccharide (LPS)-treated macrophages (e.g., M1 phenotype) mitochondrial OxPhos is shut down in favor of increased glycolysis, along with the increased synthesis of $\mathrm{FA}^{30}$. How this process impacts the bioavailability of oxylipins and their inflammatory bioactivity is unknown.

To address these questions, we examine the role of mitochondria in removing oxylipins in macrophages during inflammatory activation in vitro and in vivo, and how modulation of physiological oxylipin levels impacts leukocyte signaling relevant to an acute inflammatory challenge (macrophages, neutrophils, T cells). Genetic and lipidomic approaches characterize a metabolic $\beta$-oxidation network for oxylipins and their metabolites, during inflammation. The study identifies a mechanism for dampening oxylipin signaling that could be more broadly relevant in inflammation and infection.

\section{Results}

Inhibition of CPT1 prevents secretion of diverse peritoneal oxylipins in vivo. Mitochondrial $\beta$-oxidation of native long-chain FA requires uptake of their FA-CoA derivatives via the outer membrane transporter, CPT1, of which there are three isoforms, a,b,c. However, whether oxylipin species can be imported via CPT1 in macrophages is unknown. To test this in vivo, the pan CPT1 inhibitor etomoxir was injected intraperitoneally (i.p.) into wild-type C57BL/6 mice alone or with the bacterial lipid LPS $(1 \mu \mathrm{g})$, then peritoneal lavage harvested after $6 \mathrm{~h}$, and analyzed using LC/MS/MS. A large number of oxylipins were detected in cell-free supernatant with several showing expected elevations in response to LPS (Fig. 1a, b and Supplementary Fig. 1a). These are broadly categorized into lipids generated by $12 / 15-\mathrm{LOX}, \mathrm{COX}-1 /$ 2, or CYP450, although some are from more than one pathway (Supplementary Data File 1). Many specialist pro-resolving mediators (SPM) including resolvins or protectins were not conclusively detected. For maresin1 (Mar1, 7R,14S-diHDOHE), very small peaks were seen that fell below our limit of quantitation (LOQ) for the assay. When injecting larger amounts of sample and broadening the retention time window, two peaks were seen with one having the same retention time as the authentic standard (Supplementary Fig. 1b). It was not possible to generate a convincing MS/MS spectrum from tissues that matched the standard due to the low levels present. The detection of two isomers suggests diastereomers of 7,14-di-HDOHE, and the peaks may also contain co-eluting stereoisomers. This is suggestive of non-enzymatic origin and this peak is more appropriately named 7,14-diHDOHE.

Etomoxir stimulated small increases in several oxylipins, however when included with LPS, a far stronger impact was seen, and levels of many lipids were effectively doubled (Fig. 1a, b). This suggests that CPT1 in peritoneal mitochondria actively transports bioactive oxylipins during health, but in infection, its role is significantly enhanced.

CPT1 regulates the metabolism of endogenously generated oxylipins by peritoneal macrophages. In vivo, peritoneal oxylipins could be metabolized by mitochondria in resident macrophages, B cells and/or peritoneal membrane (mesothelium). Naive resident macrophages, which express COX-1 and 12/15-LOX (Alox15), are likely to be the primary basal source ${ }^{31}$. Thus, naive peritoneal macrophages from wild-type mice were assessed for mitochondrial removal of endogenous oxylipins. We focused on extracellular forms since most are secreted to activate GPCRs extracellularly. Serum-free media was used to prevent contamination with blood-derived oxylipins. LPS $(24 \mathrm{~h})$ stimulated prostaglandin (PG) secretion by naive resident peritoneal macrophages, consistent with COX-2 induction (Fig. 1c and Supplementary Fig. 1c). Although $12 / 15$-LOX is highly expressed in naive peritoneal macrophages, its loss in culture leads to the relatively low generation of monohydroxy FA (12-HETE and 12/15-HEPEs) (Fig. 1d and Supplementary Fig. 1d) ${ }^{32}$. Overall, oxylipin species generated by peritoneal macrophages were similar to those in lavage, although the total number/diversity was less with fewer CYP-derived species and no SPMs detected (Supplementary Fig. 1c).

Etomoxir significantly increased detected levels of several monohydroxy lipids and COX-2 products (Fig. 1c, d). This indicates that both their formation and removal is increased by inflammation, similar to in vivo (Fig. 1a, b). 12-HETE, 12-HEPE and 15-HEPE increased with etomoxir but were suppressed by LPS, likely due to inflammation-associated loss of 12/15-LOX (Fig. 1d and Supplementary Fig. 1d). Importantly, in vivo and in vitro, COX-2 and 12/15-LOX products were consistently increased by CPT1 inhibition.

Several mono- and di-hydroxy oxylipins were only detected when CPT1 was blocked, indicating that their mitochondrial metabolism exceeds their generation (Fig. 1d and Supplementary Fig. 1d). Thus, CPT1 prevents their secretion. In contrast, during 


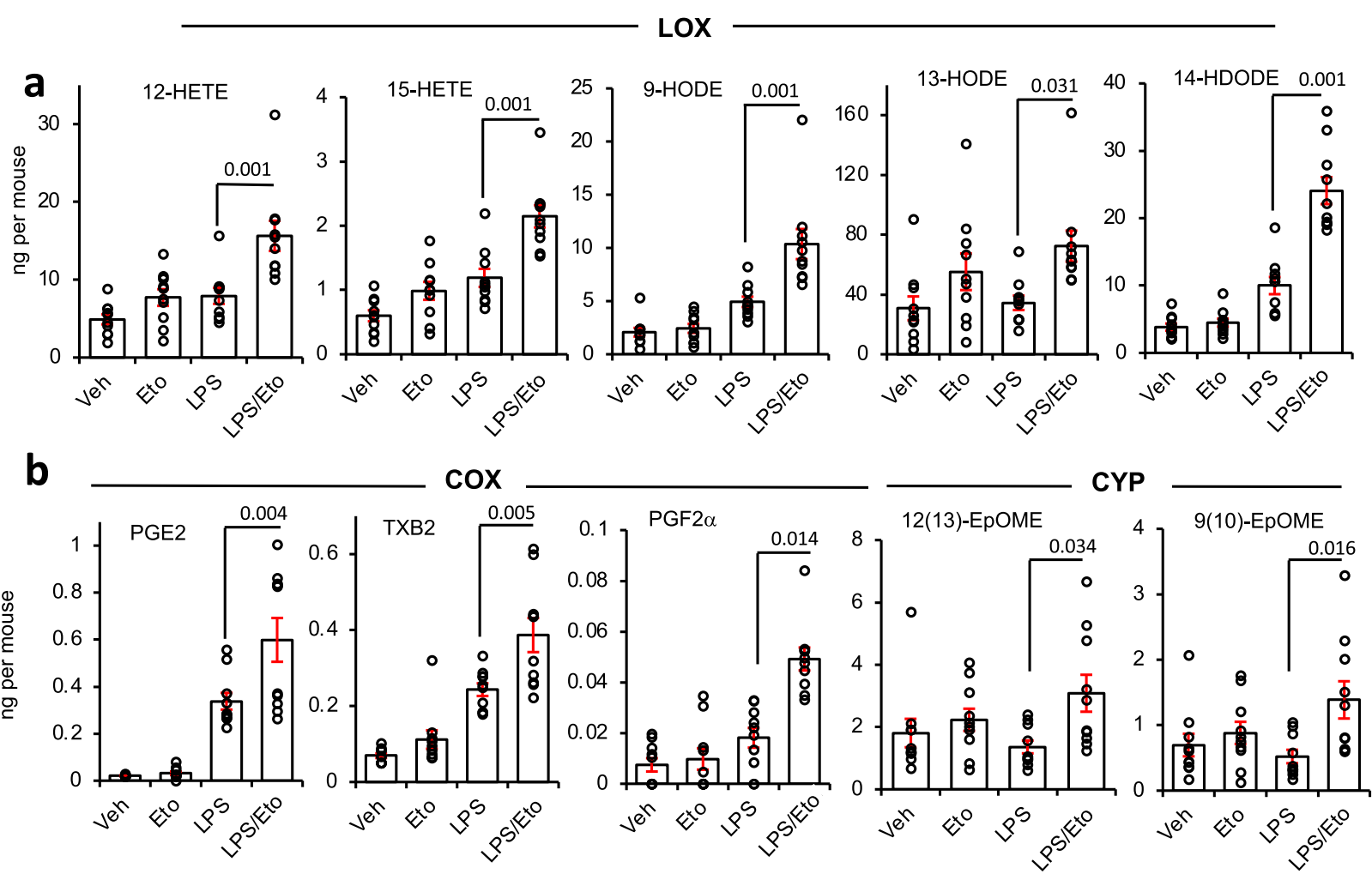

C

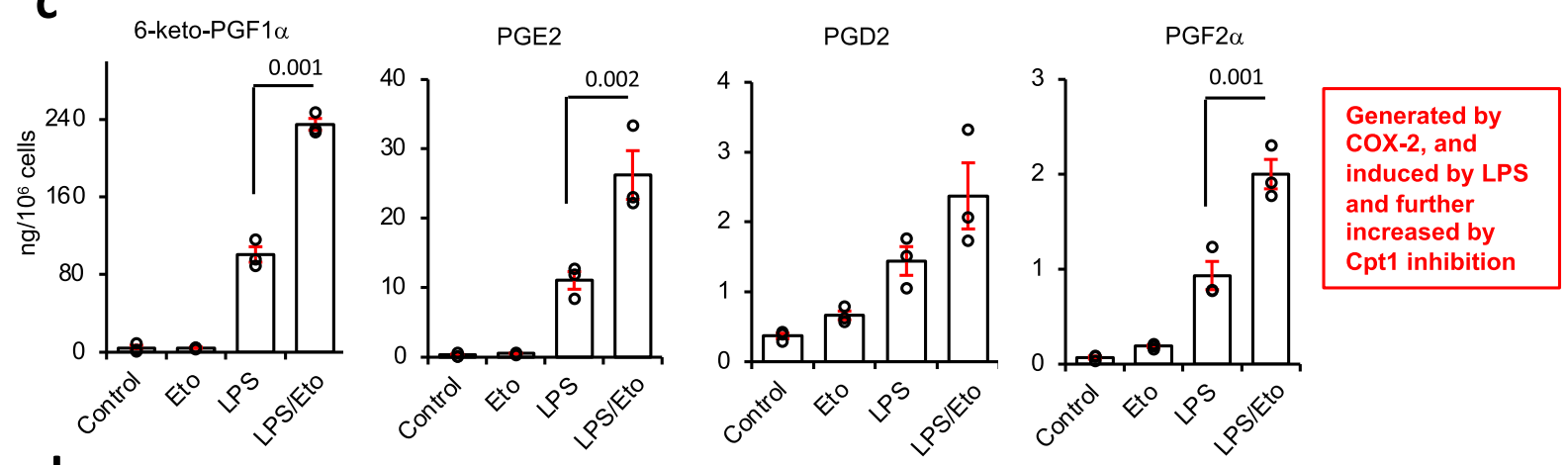

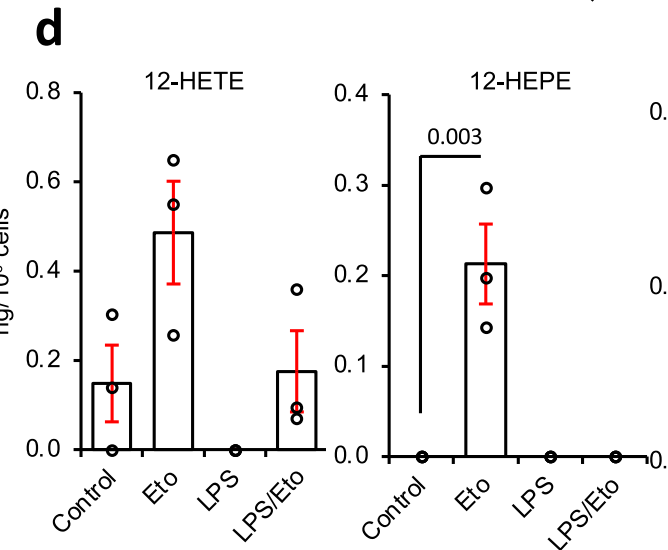

12/15-LOX: Increased by CPT-1 inhibition

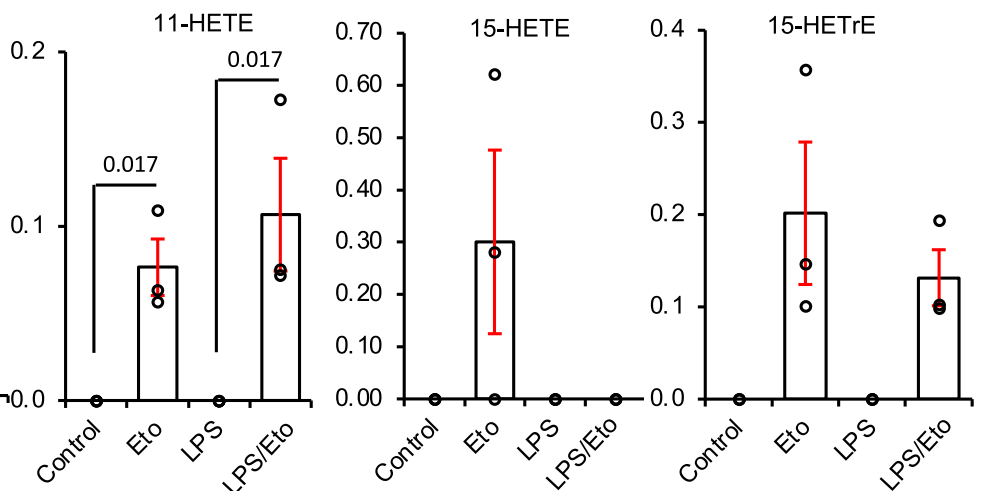

Lipids only detected with CPT-1 inhibition in peritoneal macrophages
LPS challenge, the eicosapentaenoic acid product 17,18-diHETE, generated by soluble epoxide hydrolase oxidation of CYP-derived 17,18-EET, was suppressed by etomoxir (Supplementary Fig. 1e). Overall, these data indicate that peritoneal macrophage oxylipin secretion (12/15-LOX or COX-2 derived) is counterbalanced by CPT1-mediated uptake into mitochondria. However, CYP- derived lipids show the opposite, where CPT1 inhibition dampens levels.

Bone marrow-derived macrophages (BMDM) consume diverse oxylipins from serum. Macrophages are exposed to oxylipins 
Fig. 1 Inhibition of CPT1 increases oxylipin levels significantly during inflammation in vivo and in peritoneal macrophages. a, $\mathbf{b}$ Plots for individual lipids showing that inhibition of CPT1 enhances oxylipin levels, with a bigger impact during inflammatory challenge with LPS. Wild-type mice (female, 7-9 weeks) were injected i.p. with vehicle (PBS), etomoxir $(100 \mu \mathrm{g})$, or LPS $(1 \mu \mathrm{g})$. After $6 \mathrm{~h}$, lavage was harvested and lipids extracted using SPE then analyzed using LC/MS/MS as outlined in Methods. A series of example lipids from 12/15-LOX, COX, and CYP450 are shown ( $n=10)$. c, d Plots for individual lipids showing the impact of etomoxir \pm LPS on oxylipin release by peritoneal macrophages in vitro. Peritoneal macrophages were isolated as described in Methods, then cultured in serum-free medium in the presence of etomoxir $(25 \mu \mathrm{M})$ with/without LPS (100 $\mathrm{ng} / \mathrm{ml})$. After $24 \mathrm{~h}$, the supernatant was harvested and lipids extracted using SPE, then analyzed using LC/MS/MS. A series of lipids based on enzymatic origin are shown, $n=3$ per sample. For all panels, data are mean \pm SEM, one-way ANOVA with Tukey post hoc test, stats are shown for the effect of etomoxir only, where significant. Where no bar is shown no significant difference was seen.

from other immune or stromal cells during inflammation. Serum contains numerous oxylipins from 12-LOX and COX-1, primarily monohydroxy isoforms and thromboxane, generated by white cells and platelets, but few PGs. Serum forms during innate immune responses, thus oxylipin metabolism may occur in local or systemic sites. To examine this, we tested primary murine bone marrow-derived cells differentiated to macrophages, then treated with MCSF, alone or along with LPS/IFN- $\gamma$ or IL-4 (henceforth referred to as $\mathrm{M} 0, \mathrm{M} 1$, and $\mathrm{M} 2$ ) respectively as in vitro of models for macrophage inflammation ${ }^{33}$. All macrophage phenotypes consumed significant amounts of exogenous monohydroxy oxylipins from $10 \%$ serum-containing medium, including 12-HETE/ 12-HEPE (12-LOX, generated by platelets), 5-HETE/5-HEPE (5LOX, generated by neutrophils), and 9-/13-HODEs (Supplementary Fig. 2a). 11-HETE/11-HEPE and 15-HETE/15-HEPE were removed by M0(MCSF) and M2(IL-4) cells; however, they appeared higher or unchanged for M1(LPS/IFN- $\gamma$ ) since they were simultaneously generated by COX-2, induced by LPS/IFN- $\gamma$ (Supplementary Fig. 2a). Furthermore, M1(LPS/IFN- $\gamma$ ) cells secreted large amounts of PGs and thromboxane due to COX-2, causing a net increase in their extracellular levels. (Supplementary Fig. 2a). Thus, the overall pattern was consumption of serum oxylipins by all macrophage populations, coupled with simultaneous generation of COX-2 PGs by M1(LPS/IFN- $\gamma$ ). Last, low dose etomoxir $(25 \mu \mathrm{M})$ significantly increased PGs released by M1(LPS/IFN- $\gamma$ ) cells (Supplementary Fig. 2b). Overall, this demonstrates that mitochondrial uptake of PGs suppresses their secretion from classic inflammatory BMDM cells (M1(LPS/IFN$\gamma)$ ), in the same manner as seen with naive peritoneal macrophages and in vivo with LPS peritonitis, while all BMDM cell types consume significant amounts of HETEs and HEPEs.

Dynamic control of oxylipins by CPT1 during the inflammatory challenge in RAW macrophages. Next, we used RAW cells, a model for macrophage inflammatory responses in vitro, amenable to genetic modification. These don't express 12/15LOX, therefore, to examine the impact of CPT1 on its products, cells stably overexpressing Alox15 (RAWAlox15) were generated. Basally, neither RAW ${ }^{\text {mock }}$ nor RAWAlox15 cells secreted many oxylipins, with only 9/-HODE, two PGs, and three di-hydroxy products of CYP released in low amounts (Supplementary Fig. 3a). LPS $(24 \mathrm{~h}, 100 \mathrm{ng} / \mathrm{ml})$ stimulated robust secretion of many oxylipins, including several PGs (COX-2) 9/13-HODEs, and 11 - and 15-HETEs, HETrEs and HEPEs (likely from COX-2) (Fig. 2a and Supplementary Figs. 3a, b and $4 \mathrm{a}-\mathrm{c}$ ). The primary 12/15-LOX products 12-HETE and 14-HDOHE were only detected in RAWAlox 15 cells, and their generation was increased by LPS (Fig. 2a).

CPT1 inhibition had little impact on basal oxylipin secretion (Supplementary Fig. 3a). However, it significantly elevated the LPS-dependent generation of monohydroxy FA (HETEs, HDOHEs, HETrEs, and HODEs) from RAWAlox15 cells (Fig. 2a). These data indicate that secreted oxylipins normally represent only a fraction of the overall amounts made by $12 / 15$-LOX. Thus,
LPS increases CPT1-mediated removal of 12/15-LOX products, similar to in vivo and peritoneal macrophages. Many primary $12 /$ 15-LOX products (12-HETE, 14-HDOHE) were seen, while others (9-HOTrE, 9-HODE) may be byproducts of secondary propagation. In etomoxir/LPS-treated RAWAlox15 (but not RAW cells), a lipid suggestive of resolvinD5 (RvD5, 7S,17SdiHDOHE) was detected, however of the two peaks, only one matched the retention time of the authentic standard (Supplementary Fig. 4a, b). The lipid was very low in abundance, and it was not possible to generate a reliable MS/MS spectrum from cell supernatants. As there were two peaks, they likely represent diastereomers of 7,17-di-HDOHE, and the individual peaks may also contain co-eluting stereoisomers. Thus, we labeled this 7,17diHDOHE and suggest it arises from non-enzymatic oxidation. No SPMs were detected in any other samples from RAW or RAWAlox15 cells.

Several COX-2 derived PGs and 14,15-diHETE (both CYP) were significantly decreased by etomoxir in RAW cells (Supplementary Fig. 4c). This mirrored the impact of CPT1 inhibition on peritoneal macrophage 17,18-diHETE. In summary, while LOX products were consistently elevated by etomoxir in RAW or all primary macrophages, for RAW cells alone, both COX and CYPderived lipids were suppressed.

Exogenous 12-HETE is metabolized to diene and triene tetranor metabolites via mitochondrial and non-mitochondrial $\beta$ oxidation in RAW cells. To determine the fate of oxylipins removed by CPT1, we examined the metabolism of exogenous 12-HETE by RAW macrophages. 12(S)-HETE was rapidly removed following LPS stimulation, coinciding with the formation and subsequent metabolism of two tetranor 12-HETEs (Supplementary Fig. 4d-f). These were confirmed by MS/MS as a triene, 8-hydroxy-4Z,6E,10Z-hexadecatrienoic acid (comparison with an authentic standard), and a diene, proposed as 8-hydroxy6,10-hexadecadienoic acid (through comparison with ${ }^{34}$ ) (Supplementary Fig. $5 \mathrm{a}-\mathrm{c}$ ). Both will be $8(\mathrm{~S})$ since they originate from 12(S)-HETE. As confirmation, 12(S)-HETE-d8 was added to RAW cells $(3 \mathrm{~h})$, and a deuterated diene was detected (Supplementary Fig. 5d, e). The MS/MS fragmentation of these lipids is shown (Supplementary Figs. 12-14). For the tetranor diene, fragmentation matched the expected gas-phase chemistry of hydroxylated FAs, where cleavage occurs next to the hydroxyl group (Supplementary Schemes 13 and 14). For the triene, a daughter ion at $m / z 165.1$ was seen, with high-resolution MS/MS of the standard confirming this as $\mathrm{C}_{11} \mathrm{H}_{17} \mathrm{O}$ (Supplementary Fig. 6a and Supplementary Scheme 12). NMR for the triene is shown in Supplementary Fig. 7. Almost all triene and diene were detected extracellularly, and over $4-8 \mathrm{~h}$, both disappeared from supernatant and cell pellets (Supplementary Fig. 4e, f). This suggests that macrophages generate and secrete primary and secondary metabolites of HETEs, but then re-internalize them for further metabolism. We next incubated triene tetranor 12(S)HETE with RAW macrophages, and after $3 \mathrm{~h}$, diene was detected extracellularly (Supplementary Fig. 6b). This indicates that diene 


\section{a}
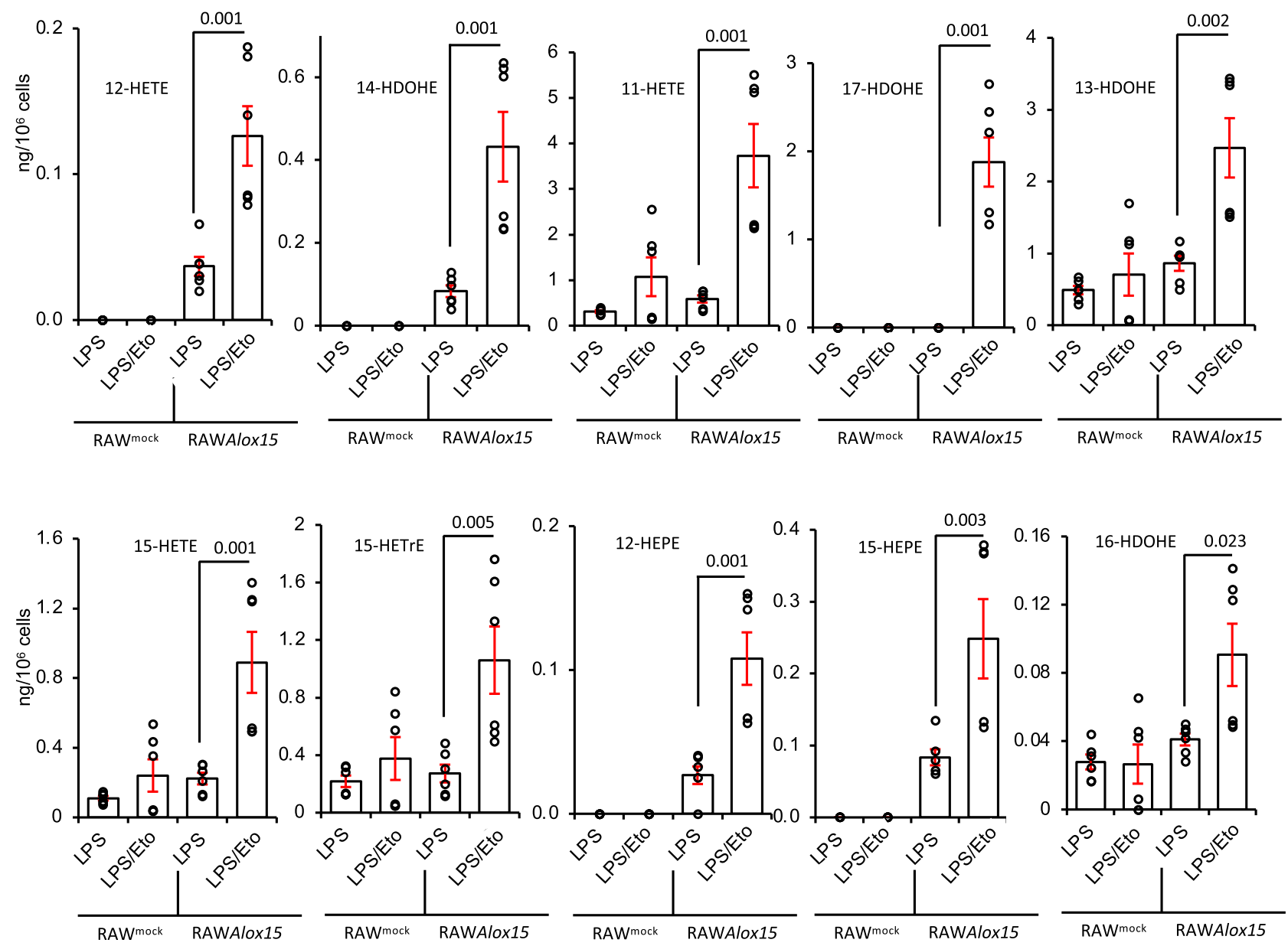

b

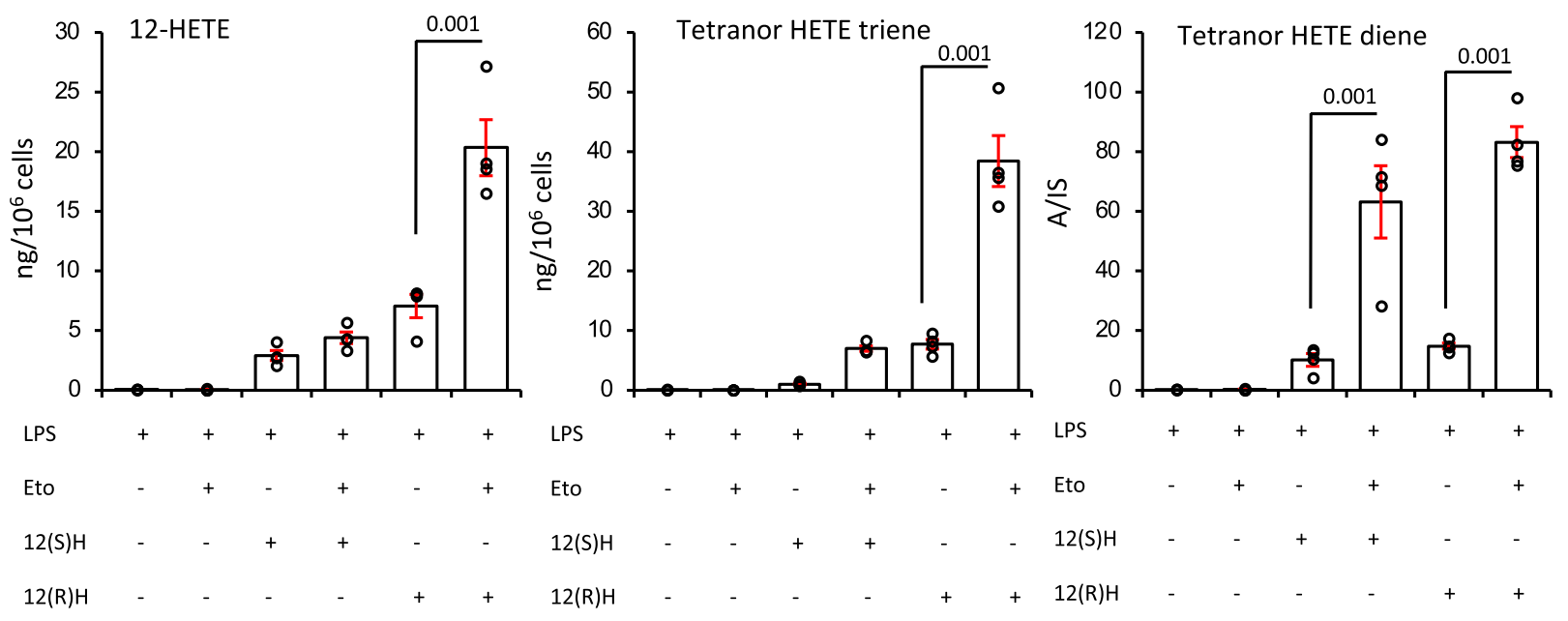

Supernatant

forms via saturation of the triene, confirming the structure as 8(S)-hydroxy-6E,10Z-hexadecadienoic acid.

Next, the impact of CPT1 inhibition on the metabolism of exogenous 12(S)-HETE or 12(R)HETE was tested. Both HETEs were rapidly removed by LPS-stimulated RAW cells, with around $0.15 \%$ or $0.4 \%$ remaining after $3 \mathrm{~h}$, for the $\mathrm{S}$ and $\mathrm{R}$ forms, respectively (Fig. 2b, left panel). Cellular and supernatant 12(S)-
HETE and 12(R)HETE were both increased by CPT1 inhibition, with 12(R)HETE most strongly impacted. Based on the impact of etomoxir, the primary removal appeared to be non-mitochondrial (Fig. 2b). 12-HETE was converted to tetranor trienes and dienes, which were detected mainly outside the cells (Fig. 2b and Supplementary Fig. 8). These were around 5-8-fold increased by CPT1 blockade, confirming that they are also dynamically 
Fig. 2 Inhibition of CPT1 increases secretion of 12/15-LOX-derived eicosanoids from RAW cells overexpressing Alox15, while RAW cells rapidly consume exogenous 12-HETE, secreting two tetranor metabolites that are themselves metabolized by mitochondrial $\boldsymbol{\beta}$-oxidation. a Blockade of CPT1 leads to a significant elevation of 12/15-LOX-derived oxylipins in RAW cells. RAWmock and RAWAlox15 cells were cultured with etomoxir (25 $\mu$ M) and/or LPS $(100 \mathrm{ng} / \mathrm{ml})$ for $24 \mathrm{~h}$ before harvest and SPE extraction of supernatant for oxylipin analysis using LC/MS/MS. Bar charts showing how oxylipins from $12 / 15$-LOX are impacted by etomoxir ( $n=6$, separate wells of cells, mean \pm SEM). b CPT1 inhibition prevents the metabolism of 12(S) or 12(R)HETE and their tetranor triene and diene metabolites. RAW cells were supplemented with $1.5 \mu \mathrm{g} 12(\mathrm{~S})$ or $12(\mathrm{R})-\mathrm{HETE} / 10^{6}$ cells for $3 \mathrm{~h}$ with/without etomoxir $(25 \mu \mathrm{M})$, then supernatants were analyzed for levels of 12-HETE and its triene and diene tetranor products using LC/MS/MS ( $n=3$ (LPS alone) or 4 (all other samples), mean \pm SEM, separate wells of cells). For all panels, comparisons are with/without etomoxir, one-way ANOVA with Tukey post hoc test, stats are shown for the effect of etomoxir only, where significant.

metabolized by mitochondria in LPS-stimulated macrophages (Fig. $2 \mathrm{~b}$ and Supplementary Fig. 8). In the case of the diene, both $\mathrm{S}$ and $\mathrm{R}$ enantiomers were similarly elevated by etomoxir treatment, while for the triene, the 12(R)HETE was most strongly impacted.

Since most removals appeared to be non-mitochondrial, we analyzed for esterification of 12(S)-HETE into phospholipid pools via Land's cycle, as previously described ${ }^{35}$. First, to determine which molecular species were formed, a precursor scanning LC/ MS/MS analysis was undertaken, scanning for negative ion precursors of $m / z 319.2$, the carboxylate anion of HETEs. Cells were supplemented with 12(S)-HETE for $3 \mathrm{~h}$, then harvested and lipid extracts were analyzed. Several precursor ions were found, between $m / z$ 738-810 that were absent in RAW cells not supplemented with 12-HETE (Fig. 3a, b). Their structures were subsequently confirmed using MS/MS, showing they originate from a series of expected HETE-containing PE or PC lipids using MS/MS scans. Next, these lipids were quantified using LC/MS/ MS. Substantial amounts of 12-HETE-PE/PC were generated during the $3 \mathrm{~h}$ incubation with 12 (S)-HETE $\left(1.4 \mu \mathrm{g} / 10^{6}\right.$ cells), totaling ca. $88 \%$ of the added oxylipin. When cells were concurrently stimulated with LPS, the proportion of HETE incorporated reduced to ca. $73 \%$, with significant reductions in levels of three lipids and trends toward a reduction in all (Fig. 3c). This identifies esterification of 12-HETE into PLs to be the dominant route of removal in RAW cells, accounting for considerably more than $\beta$-oxidation and is in line with previous work by Pawlowski et al. ${ }^{35}$. The partial dampening of this phenomenon by LPS may be a result of phospholipase activities being upregulated during inflammation. Further studies are needed to determine the impact of inflammation on all competing pathways of oxylipin removal in order to build up a more comprehensive picture of which are the dominant events under defined conditions relevant to specific situations such as inflammation, wounding, coagulation, etc.

CPT1a gene silencing confirms dysregulation of oxylipin secretion in RAW cells. Considerably higher amounts of etomoxir than we used ( $\sim 10$-fold higher) can have off-target effects due to an impact on adenine nucleotide translocase ${ }^{36,37}$. To rule this out, we targeted CPT1a using a lentiviral approach. Silencing to around $14 \%$ control levels was achieved in RAW264.7 macrophages, stably expressing shRNA that targets Cpt1a (RAW$\left.C p t 1 a^{K D}\right)$, but not those expressing a non-silencing control

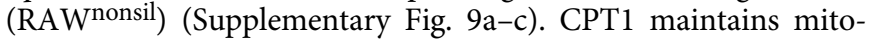
chondrial health since it imports FAs to maintain cardiolipin and phospholipid pools inside mitochondria ${ }^{38}$. Thus, CPT1-deficient cells do not proliferate normally and may alter their bioenergetic and lipid metabolism profiles. The balance between mitochondrial and peroxisomal $\beta$-oxidation and their ability to support elongase activities required to generate PUFA for oxylipin synthesis may have adapted. Similar to low dose etomoxir, the overall impact on endogenous LPS-stimulated PG synthesis in RAW cells was the suppression of supernatant and cell levels
(Figs. 3d and 4). Furthermore, the CYP450 product $(17,18$ diHETE) was suppressed, as also seen for etomoxir with naive peritoneal macrophages and RAW cells. Endogenous generation of several monohydroxy eicosanoids was suppressed by silencing of Cpt1a (Fig. 4). These data further confirm the dual role of CPT1 in both generation and removal of oxylipins depending on their enzymatic source and cell type.

Since RAW cells do not generate 12-HETE, this metabolite was exogenously added to cultured cells to evaluate mitochondrial oxylipin removal. In basal RAW cells, Cpt1 knockdown did not prevent the metabolism of 12-HETE; instead, there was a small increase, perhaps by esterification or peroxisomal metabolism (Fig. 5a). In contrast, 12-HETE removal by LPS-stimulated RAW cells was partially inhibited by Cpt1 knockdown (Fig. 5a). Furthermore, the triene and diene tetranor metabolites were significantly reduced by Cpt1 knockdown in LPS-treated but not basal RAW cells (Fig. 5b, c). This indicated that following Cpt1 knockdown, RAW cells showed reduced mitochondrial metabolism of 12-HETE coupled with the lower formation of its tetranor products post LPS stimulation.

Inflammation accelerates 12-HETE conversion to triene tetranor 12-HETE via mitochondrial $\beta$-oxidation in vivo. In vivo inhibition of CPT1 significantly elevated many oxylipins in the peritoneal cavity following the LPS challenge. To examine $\beta$ oxidation of oxylipins via mitochondria in vivo, the formation of tetranor metabolites was next measured. The diene metabolite was absent; however, tetranor 12-HETE triene was significantly elevated on CPT1 blockade during inflammatory stimulation (Fig. 5d). This confirms that inflammation in vivo not only increases the formation and mitochondrial uptake of oxylipins (shown in Fig. 1), but also their metabolism to tetranors.

Oxylipin metabolism by mitochondria is not sustaining oxidative phosphorylation (OxPhos). Mitochondrial $\beta$-oxidation of FA is directly linked with OxPhos. It forms acetyl-CoA, a substrate for the TCA cycle as well as NADH and $\mathrm{FADH}_{2}$, substrates for complexes I and II, and also transfers electrons to flavoprotein-ubiquinone oxidoreductase (ETF-QO) ${ }^{39}$. Thus, oxylipins could contribute to OxPhos. However, LPS suppresses mitochondrial respiration, through multiple mechanisms including aconitase inhibition and nitric oxide binding to complex IV, making this unlikely ${ }^{28}$. Here, LPS treatment profoundly decreased cellular respiration consistent with previous data (Supplementary Fig. 9d) ${ }^{40,41}$. Importantly, LPS did not impact mitochondrial DNA (mtDNA), or cause organelle damage as measured by DNA lesion frequency (Supplementary Fig. 9e, f). Thus, mitochondria are still present in LPS-treated cells, although $\beta$-oxidation of FAs is largely uncoupled from OxPhos.

Blocking CPT1 dampens cytokine/chemokine generation by peritoneal macrophages. CPT1 inhibition doubled the levels of PGs generated by inflammatory activated peritoneal macrophages, in 

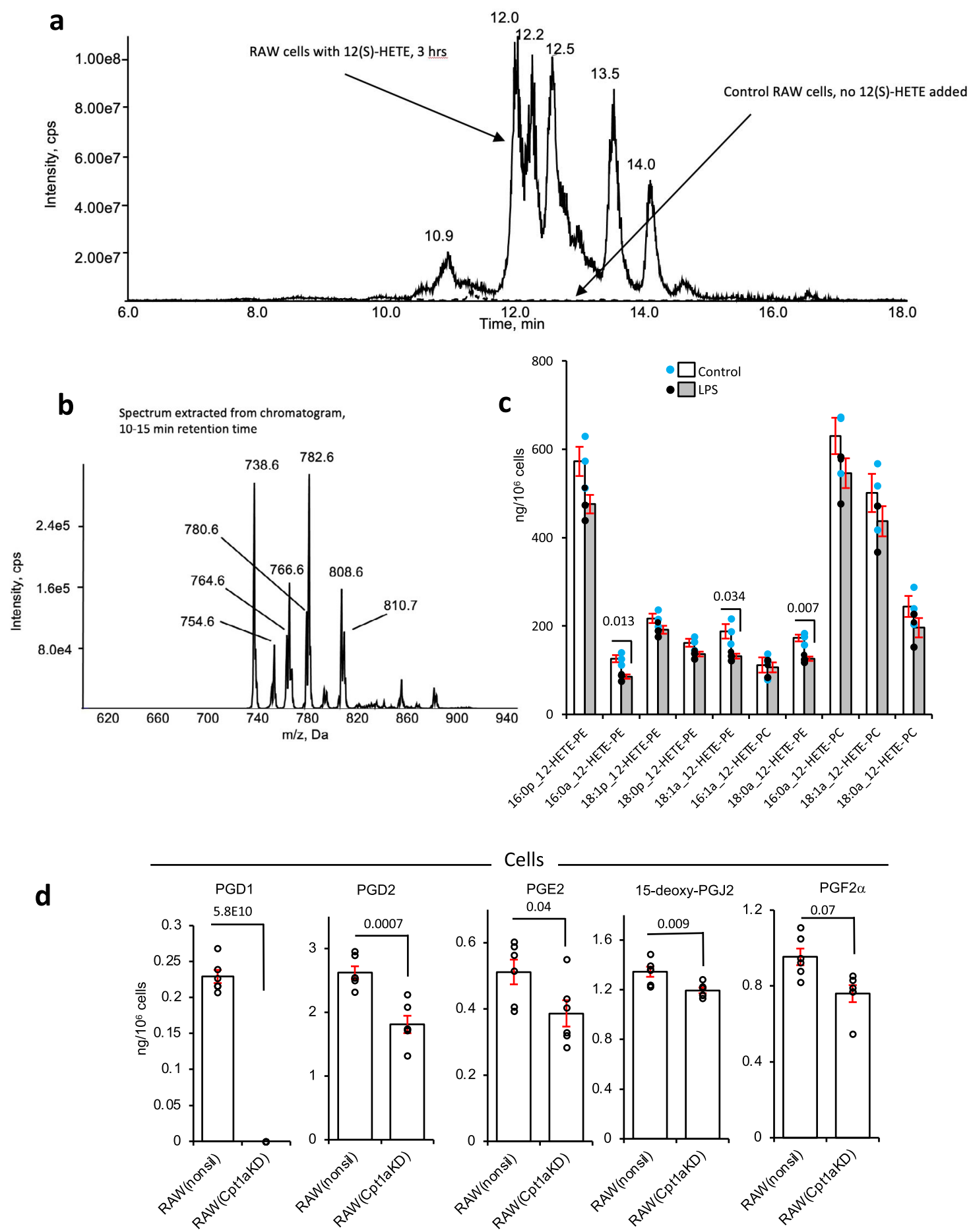

Fig. 3 Identification of phospholipid-esterified 12-HETE in RAW cells, and CPT1 knockdown reduces PG levels in RAW cells. a-c Cells (106) were incubated for $3 \mathrm{~h}$ with 12 (S)-HETE \pm LPS before lipids were extracted and analyzed as in Methods. Precursor LC/MS/MS was undertaken as described in Methods, scanning for ions that fragment to generate HETE. a Chromatogram showing elution of precursors that generate product ions with $\mathrm{m} / z$ 319.2. b MS spectrum from 10-15 min showing PE and PC species that contain 12-HETE. c Quantification of 12-HETE PE and PC species that are formed following incubation of RAW cells with $12(\mathrm{~S})$-HETE ( $n=3$, mean \pm SEM, separate wells of cells) compared with/without LPS, Student's $t$-test, two-tailed. Where no bar is shown no significant difference was seen. $\mathbf{d}$ Cpt1a knockdown dampens cellular levels of PGs in RAW cells. RAW cells expressing either the nonsilencing (RAWnonsil) or Cpt1a knockdown siRNA (RAWCpt1aKD) were treated with LPS $(100 \mathrm{ng} / \mathrm{ml})$ for $24 \mathrm{~h}$ and cell levels of PGs measured using LC/ MS/MS as in Methods ( $n=6$, mean \pm SEM, separate wells of cells). Student's $t$-test, two-tailed. Where no bar is shown no significant difference was seen. 

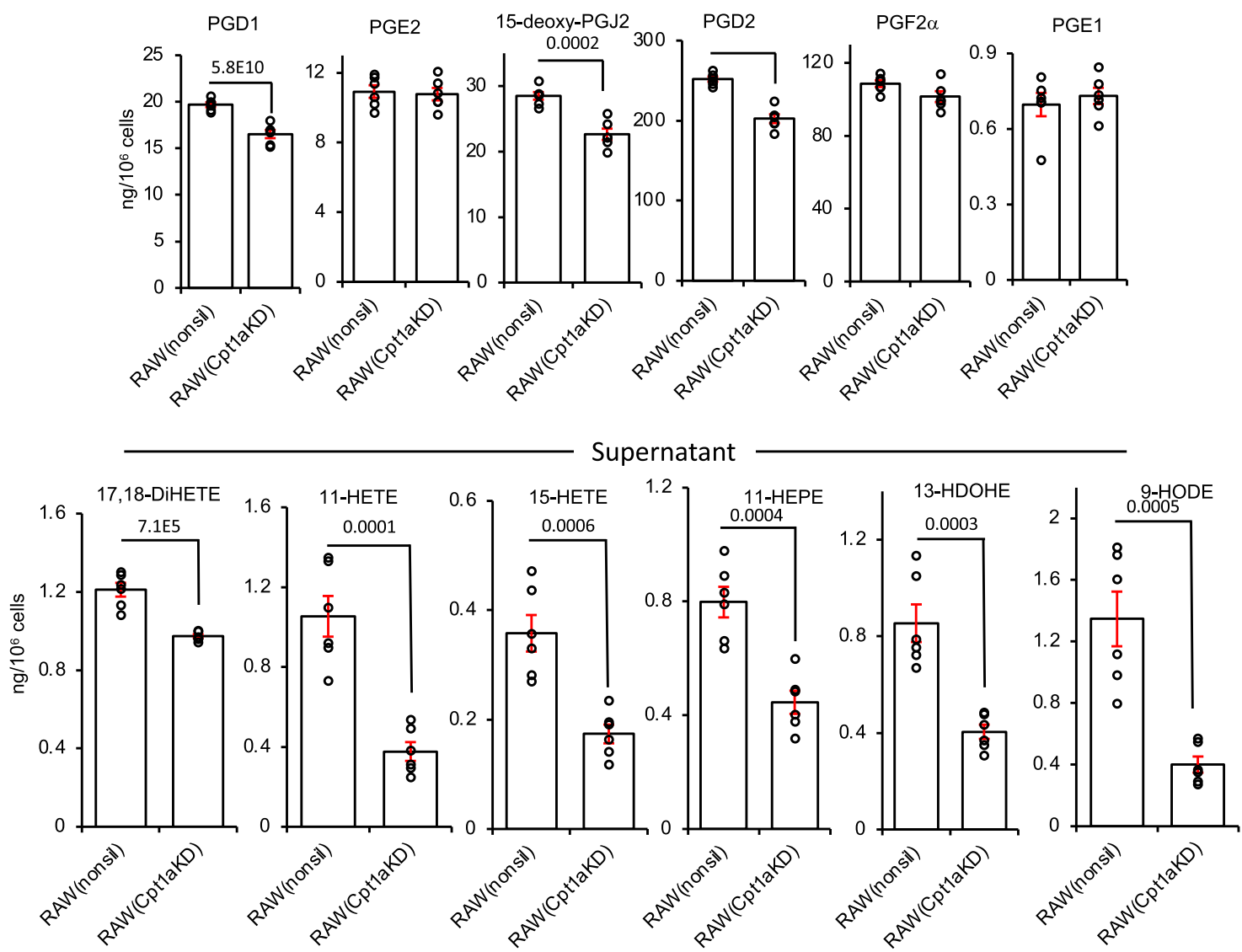

\section{Supernatant}
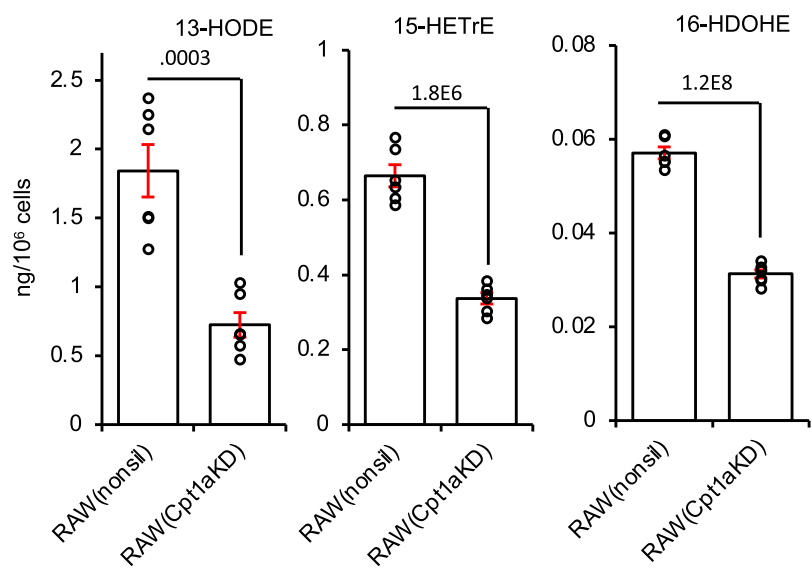

Fig. 4 CPT1 genetic knockdown reduces the secretion of oxylipins. RAW cells expressing either the non-silencing (RAWnonsil) or Cpt1a knockdown siRNA (RAWCpt7a ${ }^{K D}$ ) were treated with LPS $(100 \mathrm{ng} / \mathrm{ml})$ for $24 \mathrm{~h}$ and secretion of PGs, 17,18-diHETE, or monohydroxy oxylipins were measured using LC/MS/ MS as in Methods ( $n=6$, mean \pm SEM, separate wells of cells). Student's $t$-test, two-tailed. Where no bar is shown no significant difference was seen.

particular the abundant prostacyclin $\left(\mathrm{PGI}_{2}\right)$ metabolite 6-keto$\mathrm{PGF}_{1} \alpha$ and $\mathrm{PGE}_{2}$ (Fig. 1c). Thus, we next sought to examine whether this impacted PG-mediated autocrine signaling. Several studies have reported that oxylipins such as monohydroxy FAs, $\mathrm{PGE}_{2}$ or iloprost ( $\mathrm{PGI}_{2}$ analog) can suppress cytokine and chemokine generation in monocytes or macrophages, including by EP receptor signaling, or PPAR- $\gamma$ activation ${ }^{42-46}$, thus we tested the impact of etomoxir on generation of TNF or RANTES in LPSstimulated peritoneal macrophages. For both proteins, there was a significant decrease in the generation when etomoxir was present (Fig. 5e, f). This supports the idea that removing oxylipins can dampen their signaling in macrophages.

Oxylipin levels detected in vivo regulate leukocyte responses in a concentration-dependent manner. Oxylipins are primarily secreted to act on other cell types in a paracrine manner. Next, to address whether macrophage mitochondrial $\beta$-oxidation impacts wider oxylipin signaling between other cell types, human 
a

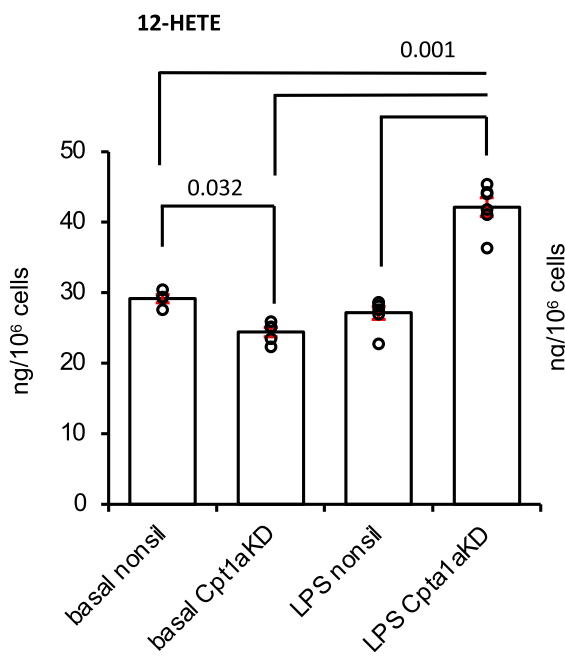

d
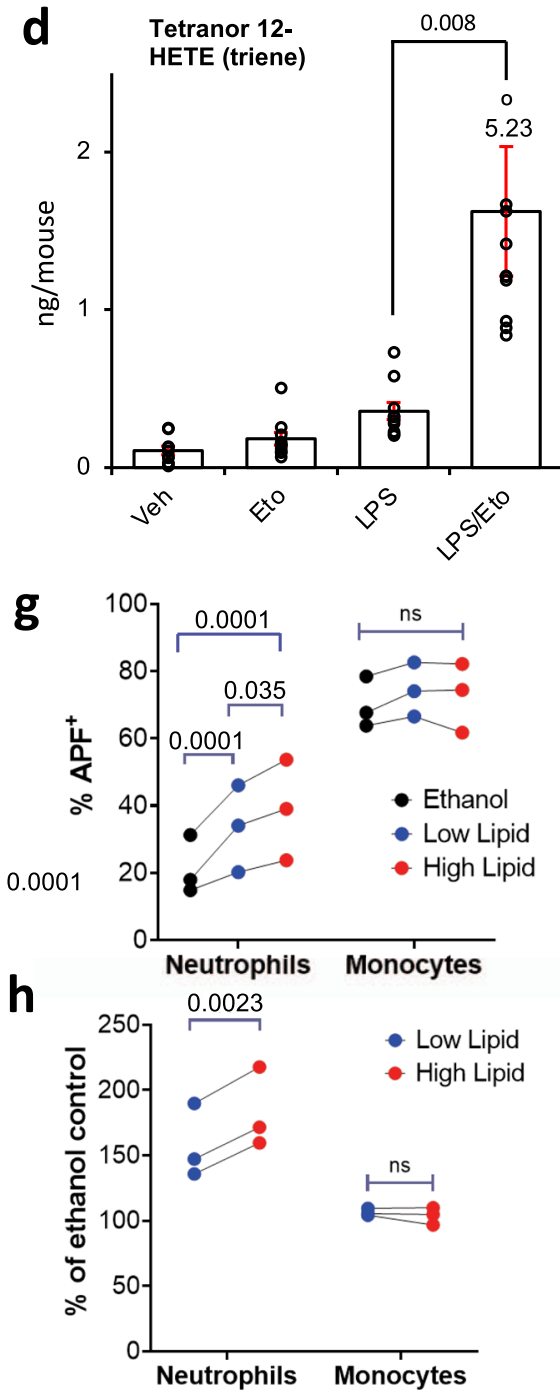

b

Tetranor 12-HETE (triene)

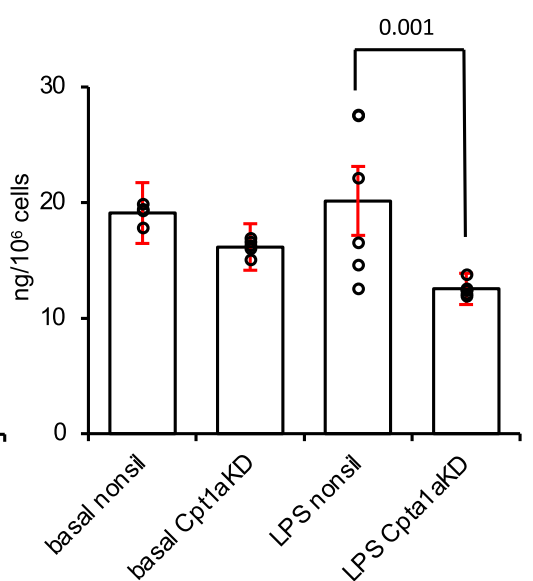

C

Tetranor 12-HETE (diene)

e
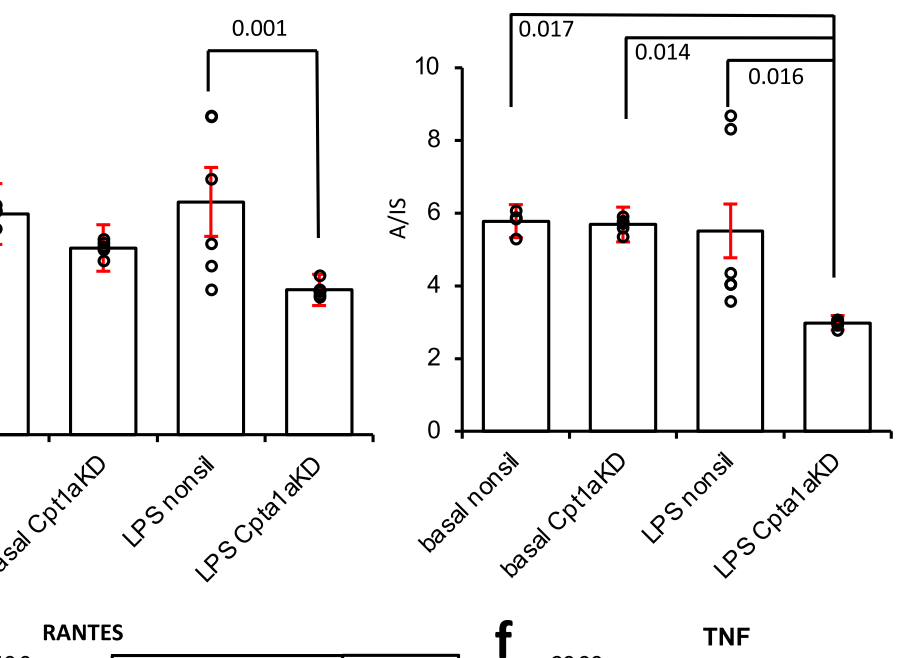

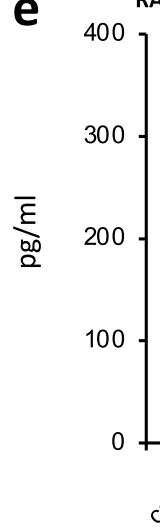
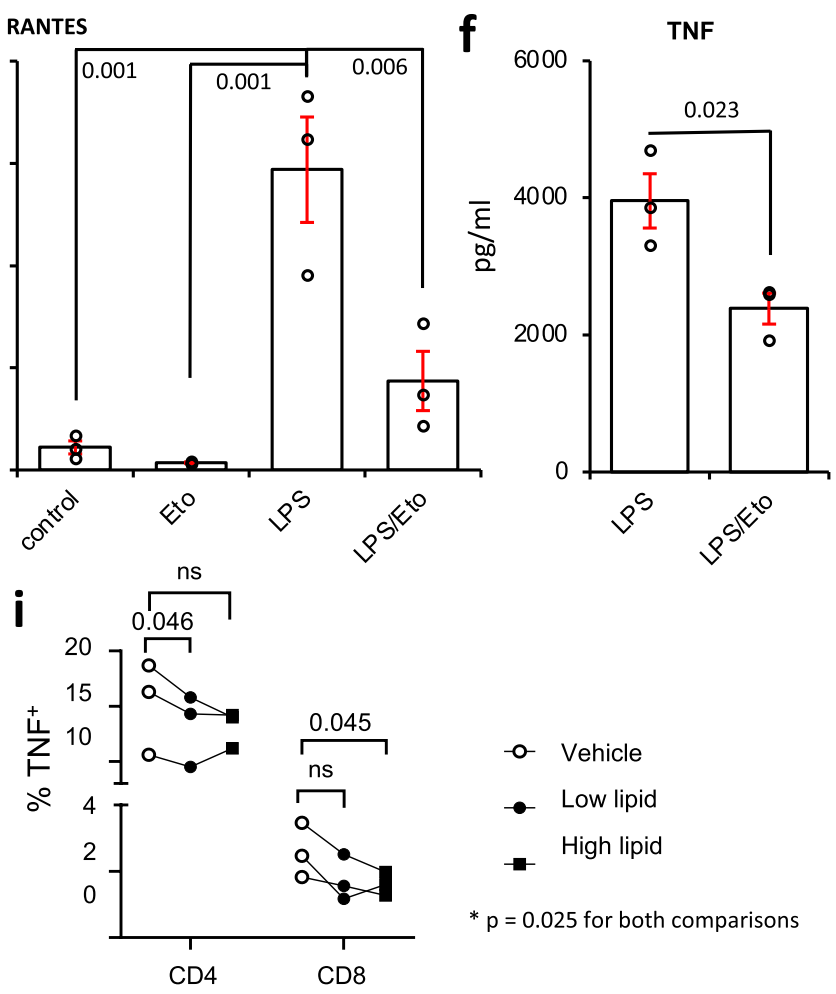

-o Vehicle

$-\quad$ Low lipid

$\rightarrow$ High lipid

$* p=0.025$ for both comparisons

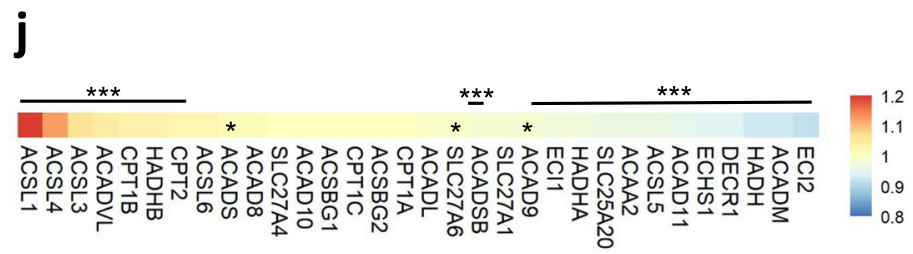

Human blood expression of genes, infected versus healthy subjects

phagocyte and T-cell responses to these lipids were determined. Neutrophils were chosen since they are known to be sensitive to $\mathrm{PGE}_{2}$, via EP receptor signaling ${ }^{47,48}$, while $\mathrm{T}$ cells can be regulated by PPAR $\gamma$, a transcription factor that is regulated by many LOX-derived oxylipins ${ }^{49-55}$. Here, reactive oxygen species (ROS) production elicited by Staphylococcus epidermidis, a common pathogen in human peritonitis was measured by aminophenyl fluorescein (APF) fluorescence of whole blood leukocytes ${ }^{56}$. Two concentrations were used that represented amounts of 52 individual oxylipins detected in vivo, in the presence or absence of etomoxir (with LPS) (Supplementary Table 1). Here, ROS generation by neutrophils was consistently enhanced by higher or 
Fig. 5 Cpt1a genetic knockdown modulates the metabolism of 12-HETE and its metabolites by RAW cells, etomoxir reduces the metabolism of tetranor triene in vivo, and prevents LPS stimulation of RANTES/TNF $\alpha$ generation, oxylipins regulate leukocyte responses, and transcriptomics of human neonatal sepsis proposes multiple genes that support a mitochondrial oxylipin $\boldsymbol{\beta}$-oxidation pathway. a-c Knockdown of Cpt1a alters $12-\mathrm{HETE}$

metabolism. RAW cells were incubated \pm LPS $(100 \mathrm{ng} / \mathrm{ml}$, for $4 \mathrm{~h})$ with 12-HETE $(2.34 \mu \mathrm{g} / \mathrm{ml})$ added after the first hour (thus added for $3 \mathrm{~h}$ ). Supernatants were harvested, then lipids were extracted and analyzed for 12-HETE and metabolites using LC/MS/MS as described in Methods ( $n=4$ (basal nonsil), 5 (basal Cpt1aKD), or 6 (other groups), separate wells of cells, mean \pm SEM). $\mathbf{d}$ Tetranor triene 12-HETE is increased in vivo during inflammation with CPT1 inhibition. Wild-type mice (female, 7-9 weeks) were injected i.p. with vehicle (PBS), etomoxir (100 $\mu \mathrm{g})$, and/or LPS ( $1 \mu \mathrm{g})$. After $6 \mathrm{~h}$, lavage was harvested and lipids extracted and analyzed using LC/MS/MS as outlined in Methods ( $n=10$, individual mice). Outliers are shown as red, or with values. e, $\mathbf{f}$ Etomoxir dampens the generation of RANTES and TNF by peritoneal macrophages. Supernatants from macrophages cultured in vitro for $24 \mathrm{~h}$ with LPS $(100 \mathrm{ng} / \mathrm{ml})$ /etomoxir $(25 \mu \mathrm{M})$ were tested for RANTES/CCL5 or TNF using ELISAs as described in Methods ( $n=3$ per group, separate wells of cells, mean \pm SEM). a-f ${ }^{\star} p<0.05,{ }^{\star \star} p<0.01,{ }^{\star \star \star} p<0.005$ with/without etomoxir, one-way ANOVA with Tukey post hoc test was used. $\mathbf{g}$, $\mathbf{h}$ Oxylipin levels detected in vivo promote neutrophil ROS generation. Whole blood was incubated with oxylipin mixtures (Supplementary Table 1), and then ROS generation in response to stimulation using opsonized S. epidermidis was determined by APF fluorescence as indicated in Methods. Three individual donor samples were separately tested. Monocytes and neutrophils were analyzed using the gating strategy as outlined in Methods. Response to high or low oxylipin doses was expressed either as \%APF + cells or \% of the vehicle (ethanol) response. Each donor is shown separately. Data were analyzed by repeated-measures two-way ANOVA (g-pairing $p<0.0001$, cell type $p=0.018$, lipid $p=0.0002$, and interaction $p=0.0005$; $\mathbf{h}-$ pairing $p=0.0008$, cell type $p=0.0094$, lipid $p=0.0082$, and interaction $p=0.0024$ ), Sidak's post hoc tests are indicated $-\mathbf{g}$ Neutrophils ethanol vs low lipid $p=0.0001$, neutrophils ethanol vs high lipid $p<0.0001$, neutrophils low lipid vs high lipid $p=0.0350$, monocytes ethanol vs low lipid $p=0.7020$, monocytes ethanol vs high lipid $p=0.8075$, monocytes low lipid vs high lipid $p=0.8075-\mathbf{h}$ neutrophils low lipid vs high lipid $p=0.0023$, monocytes low lipid vs high lipid $p=0.2363)$. i Oxylipin levels detected in vivo modulate CD4+ and CD8+ T-cell activation. Whole blood was incubated with oxylipin mixtures

(Supplementary Table 1), and then generation of TNF was determined in response to activation using CD3/CD28 beads as indicated in Methods. CD8+ and CD4+ T cells were analyzed using the gating strategy outlined in Methods. Data from individual donors is shown. Two-way ANOVA (repeated measures) was used, pairing $p=0.0039$, interaction $p=0.1690$, cell type $p=0.0007$, effect of lipid $p=0.0253$, Sidak's post hoc tests are indicatedCD4: no lipid vs low lipid $p=0.0461$, CD4: no lipid vs high lipid $p=0.7115$, CD4: low lipid vs high lipid $p=0.9989$, CD8: no lipid vs low lipid $p=0.3742$ CD8: no lipid vs high lipid $p=0.0449$, CD8: low lipid vs high lipid $p=0.9952)$. j Human transcriptomics data show significant upregulation of several genes including ACSL and CPT isoforms. Gene expression (log2 expression data) from 35 controls and 26 bacterial confirmed sepsis cases published in 57 were compared for expression of 32 genes putatively involved in mitochondrial import and $\beta$-oxidation of oxylipins, based on known metabolic pathways for long-chain native FA. ${ }^{\star} p<0.05,{ }^{\star \star \star} p<0.005$, Student's $t$-test, two-tailed, then adjusted using Benjamini-Hochberg test.

lower oxylipin levels. However, there was a significant concentration-dependent impact in three individual donors (Fig. 5g, h). Thus, the higher amount, consistent with levels detected during CPT1 inhibition, was significantly more potent at activating neutrophils. Monocyte ROS generation was unaffected at either dose (Fig. 5g, h). Next, T-cell responses in peripheral blood mononuclear cell (PBMC) were determined by measuring TNF generation following TCR-specific stimulation of CD4+ and CD8 + T-cell populations. Here, we found that in general oxylipins had a statistically significant suppressive effect on TNF production in both subsets. Post-test analysis revealed that the lower level of oxylipins alone significantly suppressed TNF in CD4 + cells. In the case of CD8 + cells, only the high level showed significance, while there was a trend at both doses (Fig. 5i). Taken together, these pilot data indicate that modulation of oxylipin levels, within physiological amounts detected in vivo can regulate immune responses of neutrophils and $\mathrm{T}$ cells in vitro, supporting the concept that oxylipin removal by mitochondria during inflammation could regulate broader cellular innate immune responses.

Transcriptional analysis of human neonatal bacterial sepsis identifies gene candidates responsible for mitochondrial oxylipin metabolism. The enzymes that import oxylipins into mitochondria are not conclusively known, but it is very likely that those importing long-chain FA are involved. These include five acyl-CoA synthetase long-chain family members (ACSL1,3-6), as well as CPT1a, $1 b, 1 c$, and $C P T 2$. The gene products mediate the formation of $-\mathrm{CoA}$ and then -carnitine derivatives that are required for long-chain FA uptake across mitochondrial membranes (Supplementary Fig. 10 and Supplementary Table 2). This is followed by mitochondrial $\beta$-oxidation, which catalyzes sequential removal of 2-carbon fragments to generate acetyl-CoA, and chain shortened metabolites. Genes that encode proteins that metabolize long-chain PUFA include HADHA, HADHB, ECI1,
DECR1, and ACADVL (Supplementary Fig. 15 and Supplementary Table 2$)^{22}$. A set of 33 genes were compiled including also isoforms with a preference for medium or shorter chain FA. Their expression during human infection in vivo was tested using a microarray from human neonatal whole blood cells (35 cases, 26 controls). Here, infants suspected of infection had blood cultures conducted, and a diagnosis of bacterial sepsis was confirmed ${ }^{57}$. Out of the genes tested, 22 were significantly different with 9 upand 13 downregulated in sepsis. Notably, several upregulated genes encode proteins involved in the generation of long-chain FA-CoAs and their interconversion to acyl-carnitines in mitochondria (Figs. 5j and 6a). For the most relevant, seven were significantly upregulated (ACSL1,3,4,CPT1b, CPT2, ACADVL, $H A D H B)$ while three were downregulated (ACSL5, ECI1, $H A D H A)$ (Figs. 5j and 6a). This indicates a high degree of regulation during complex bacterial infection in seriously ill humans, suggesting the specific isoforms that may be involved in oxylipin removal. We also interrogated transcriptomic data from stromal tissues extracted from mice challenged (i.p.) with a cell-free supernatant from a clinical isolate of S. epidermidis (SES $)^{58}$. Here, there was overall upregulation, showing a similar trend to the human bacterial dataset (Fig. 6b, c). Although most individual genes were not statistically significant, Cpt1a showed around 2 -fold induction, which was significant $6 \mathrm{~h}$ post infection.

Consistent upregulation of ACSL isoforms is revealed across multiple human and murine macrophage datasets, and inhibition of ACSLs dampens 12-HETE metabolism by $\beta$-oxidation in RAW cells. Next, data from three mouse BMDM or three human PBMCs studies were downloaded from GEO $^{59-64}$. Several isoforms of ACSL were found to be upregulated, including ACSL1,3,4 (human study) or Acsl1,5 (SES peritonitis and in vitro BMDM experiments). Notably, ACSL1 expression was upregulated in all studies (Figs. 6d, e and 7a, b). For BMDM, Acsl1 was consistently significantly induced on stimulation using either 
a
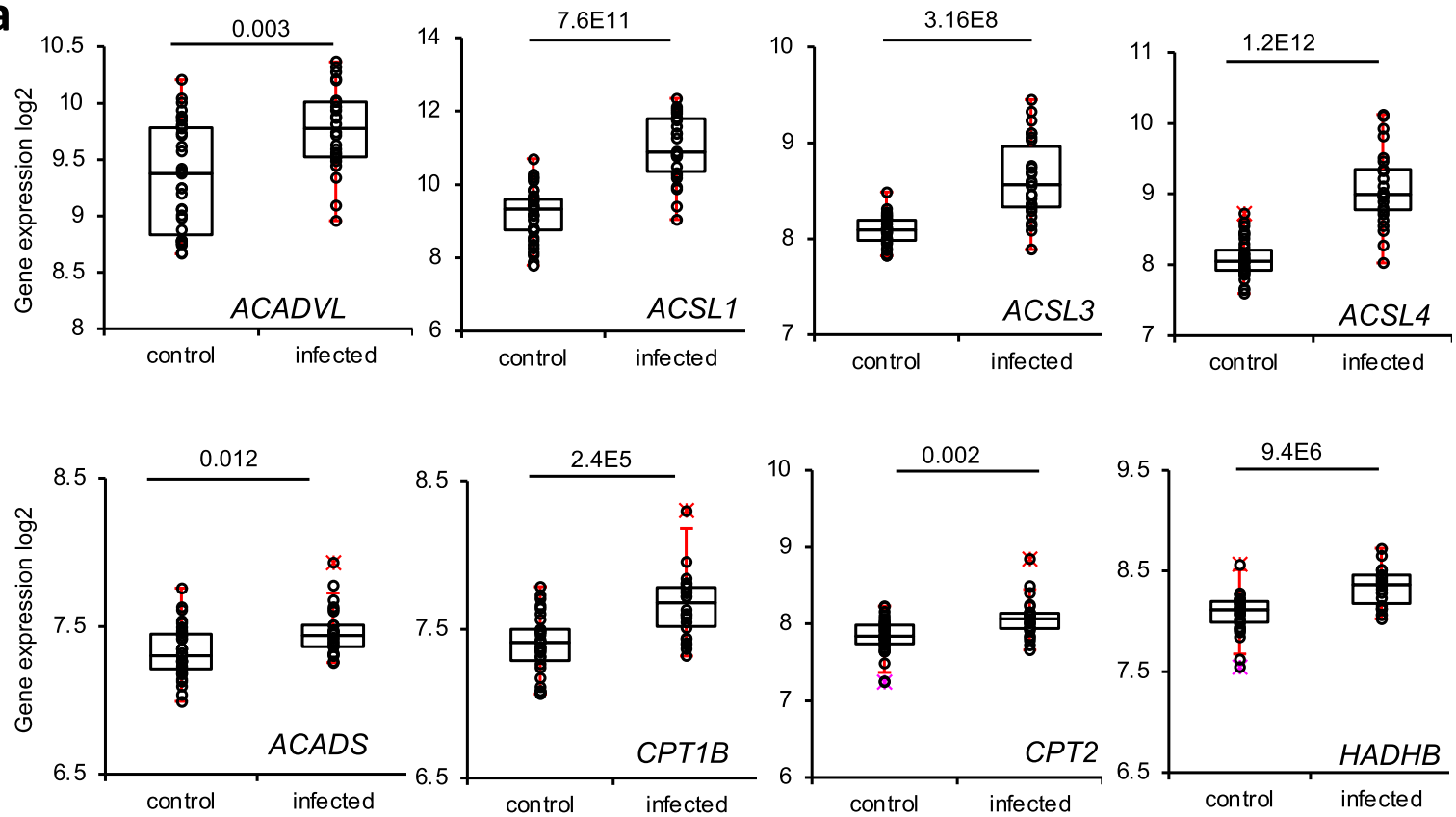

b

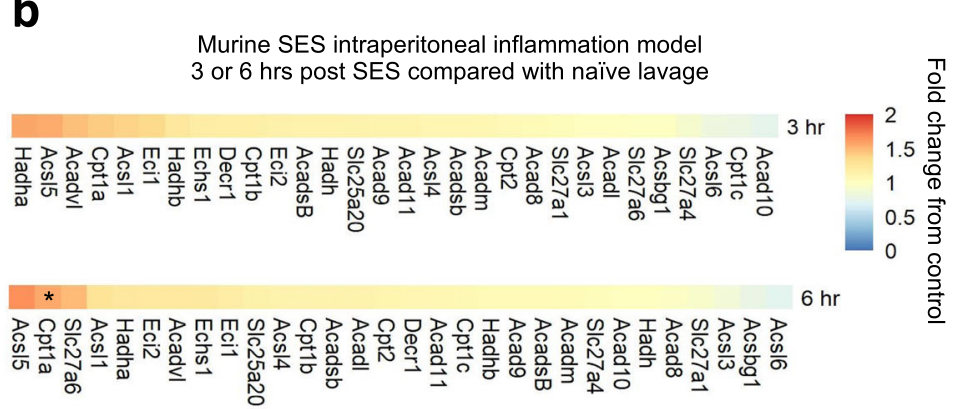

C
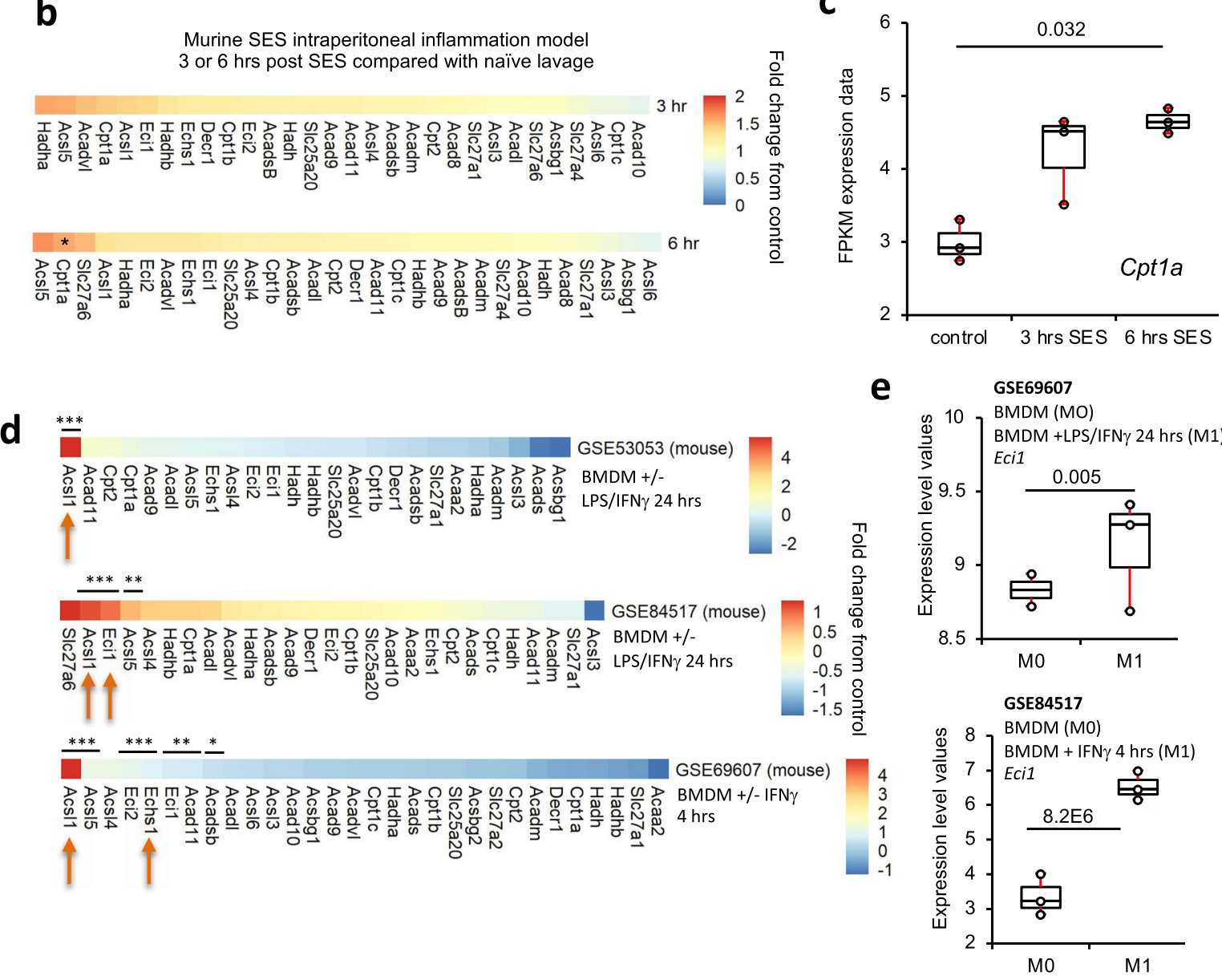

LPS/IFN- $\gamma$ or IFN- $\gamma$ alone (Figs. $6 \mathrm{~d}$ and 7a). Two of the mouse BMDM datasets also indicated significant increases in Eci1, encoding 3,2-enoyl-CoA isomerase (Supplementary Fig. 15 and Fig. 6d, e). In human studies, ACSL1 was also induced by LPS/ IFN- $\gamma$ or IFN- $\gamma$ (Fig. 7b). To confirm the functional relevance of ACSL upregulation, the impact of the pharmacological inhibitor
Triascin C on 12-HETE metabolism was tested. 12-HETE removal was strongly inhibited while the formation of tetranors was dampened between 60 and 95\% (Fig. 7c). This is consistent with the requirement for CoA-esters for 12-HETE $\beta$-oxidation (mitochondrial or peroxisomal), and confirms the involvement of ACSLs. We note that 12-HETE uptake into phospholipid pools 
Fig. 6 Transcriptomics of the mitochondrial $\beta$-oxidation pathway reveals ACSL1/Acs/1 as a key checkpoint response to LPS inflammation in human and mouse macrophages. a Plots for the eight significantly upregulated genes in the human neonatal dataset are shown. $n=35$ and 26 for infection and controls, respectively. Student's t-test, two-tailed, then adjusted using Benjamini-Hochberg test. b, c Transcriptomics of mouse peritonitis shows significant upregulation of Cpt1a at $6 \mathrm{~h}$ post SES. Gene expression data from peritoneal membranes harvested post SES challenge were analyzed for expression of 32 genes ( $n=3$ per group) ${ }^{\star} p<0.05$, Student's $t$-test, two-tailed, adjusted using Benjamini-Hochberg test. c The plot for Cpt1a expression. d, e Transcriptomics reveals Ascl1 and Eci1 to be highly upregulated in response to LPS/IFN- $\gamma$ in murine BMDM. Transcriptomics data obtained from GEO database were analyzed as outlined in Methods for expression of 34 genes selected for potential or known involvement in mitochondrial $\beta$-oxidation. Samples were BMDM treated with either LPS/IFN- $\gamma$ or IFN $\gamma$ alone as indicated. For all genes, the log2fold change was calculated and plotted using Pheatmap in R (d). e Box and whisker plots for normalized expression (using Limma and Oligo Bioconductor packages) of Eci7 in mouse datasets with $n=3$ for all groups, adjusted using Benjamini-Hochberg test ( $n=3$ for all groups except for GSE53053 MO $(n=2)$ ). Box shows median, and interquartile ranges $(I Q R)$. The ends of the whisker are at $1.5 \times I Q R$ above the third quartile (Q3) and $1.5 \times I Q R$ below the first quartile (Q1). If minimum or maximum values are outside this range, then they are shown as outliers.

via Land's cycle esterification also requires ACSL activity. Last, we saw a small non-significant suppression of 12-HETE removal by LPS in RAW cells (Fig. 7c). This is consistent with the process being mainly via Land's cycle esterification, which was in turn slightly suppressed by LPS (Fig. 3a-c).

Arachidonate (20:4) levels increase in response to inflammation and are dampened by CPT1 activity. Pharmacological or genetic inhibition of CPT1/Cpt1a dampened PG generation in RAW cells (Figs. 3d and $4 \mathrm{a}$ and Supplementary Fig. 4c). As a potential explanation, we considered whether LPS-driven inflammation leads to elevated PUFA biosynthesis, which is then dampened by CPT1 inhibition. Specifically, $\beta$-oxidation generates acetyl-CoA, which is converted to malonyl-CoA, used to chain elongate and desaturate essential FA to form PUFA via the gene products of ELOVL and FADS. To examine this, the impact of LPS/etomoxir on saturated FA (e.g., 16:0, 18:0) and PUFA (e.g., 20:4) levels was tested. No significant effect was seen for saturated FA, although etomoxir tended to decrease them following LPS treatment (Fig. 7d). In contrast, LPS stimulated significant increases in 20:4 levels. Etomoxir had opposing effects on this, dampening AA in resting cells, while enhancing its levels during LPS stimulation (Fig. 7d). Here, LPS most likely increases 20:4 by promoting its phospholipase $\mathrm{A}_{2}\left(\mathrm{PLA}_{2}\right)$-dependent release from PL membranes. Some 20:4 is then dynamically removed via $\mathrm{CPT} 1$, and thus is sensitive to etomoxir. In contrast, the negative impact of etomoxir in resting cells may relate to the reduction in the supply of malonyl-CoA for elongation. Importantly, the impact of etomoxir on 20:4 was only seen during LPS stimulation, consistent with upregulation of ACSLs and accelerated oxylipin consumption we observed during inflammation. Last, the impact of inflammatory activation on FADS or ELOVL expression was examined, using the same GEO datasets tested above. However, no consistent trends were seen for any isoforms (data not shown). This indicates that the elongation pathway is not upregulated at the gene expression level by inflammation. In summary, the elevation of 20:4 following inflammatory activation most likely arises from activities of phospholipases, and CPT1 then plays a role in its subsequent removal. However, we were unable to elucidate the reason for the suppression of PG biosynthesis by inhibition of CPT1, and further studies are required.

\section{Discussion}

Oxylipins are soluble mediators generated during inflammation and infection that link lipid metabolism with immunity. Dynamically controlling their removal is essential to dampen inappropriate inflammation. Here we show that blocking the mitochondrial enzyme CPT1, effectively doubles secreted levels of endogenously generated oxylipins in vivo during the inflammatory challenge. Using exogenous 12-HETE, we propose a paradigm in which macrophage removal of oxylipins and their metabolites involves cycles of secretion and re-internalization of partially oxidized tetranor intermediates, followed by further mitochondrial metabolism. Extending previous studies, we found high levels of secreted free acid tetranors in vitro and in vivo, thus regardless of whether formed in peroxisomes or mitochondria, these lipids appear to undergo repeated cycles of acylation/deacylation (to CoA and carnitine forms, via ACSLs and CPT) facilitating their rapid secretion, re-internalization and further $\beta$ oxidation $^{34,65}$. This demonstrates a complex cellular metabolism for tetranors, and raises questions as to why they are secreted, instead of being simply metabolized to terminal end products. Tetranor compounds can only be formed via $\beta$-oxidation (either peroxisomal or mitochondrial). For this to occur, the lipids must be taken up by the cells. Transport of oxylipins into cells is long known to be mediated by a combination of diffusion, and in the case of PGs, prostaglandin transporters such as the protein encoded by SLCO $2 \mathrm{~A} 1^{66}$. These proteins have for decades been considered important for supporting the cellular inactivation of individual PG species, and their role in tetranor metabolism could be explored.

Our finding that oxylipin metabolism is strongly stimulated by LPS has implications for the rare genetic disorder, CPT1 deficiency, where symptomatic episodes and death are triggered by acute infection ${ }^{67}$. Also, rates of hospitalization and deaths in infants with the CPT1a variant p.P479L are higher for homozygotes with respiratory, dental, and aural infections ${ }^{68,69}$. This variant reduces CPT1a activity by around $50 \%{ }^{68,69}$. The observation that infection causes severe symptoms indicates that mitochondrial $\beta$-oxidation is a protective response to pathogen challenge. This is in line with our findings that CPT1-dependent oxylipin removal is elevated by the bacterial product LPS, and suggests that this may be an important part of the physiological response to infection, functioning as an "off-switch" for pathologic signaling. Whether oxylipin levels are elevated in patients with CPT1a deficiency is not known, and future studies to examine this are warranted.

Mitochondrial uptake of short-/medium-chain native FAs is independent of CPT1; however, long-chain native FA require first activation to acyl-CoA derivatives by acyl-CoA synthetases (ACSL), then conversion to acyl-carnitines, via CPT1 (Supplementary Fig. 10). Following import into mitochondria via an inner membrane transporter, FA are re-converted to acyl-CoAs by CPT2. We note that so far, evidence for CPT1 being able to import oxylipins relies on the use of etomoxir and Cpt1a silencing. Due to the lack of availability of oxylipin-CoAs, we are as yet unable to directly test for enzyme activity with isolated mitochondria. Acyl-CoAs are used for mitochondrial $\beta$-oxidation, with sequential removal of two $C$ fragments, and generation of acetyl-CoA. Using a human sepsis cohort and multiple human and murine in vitro models, we propose a biochemical pathway 

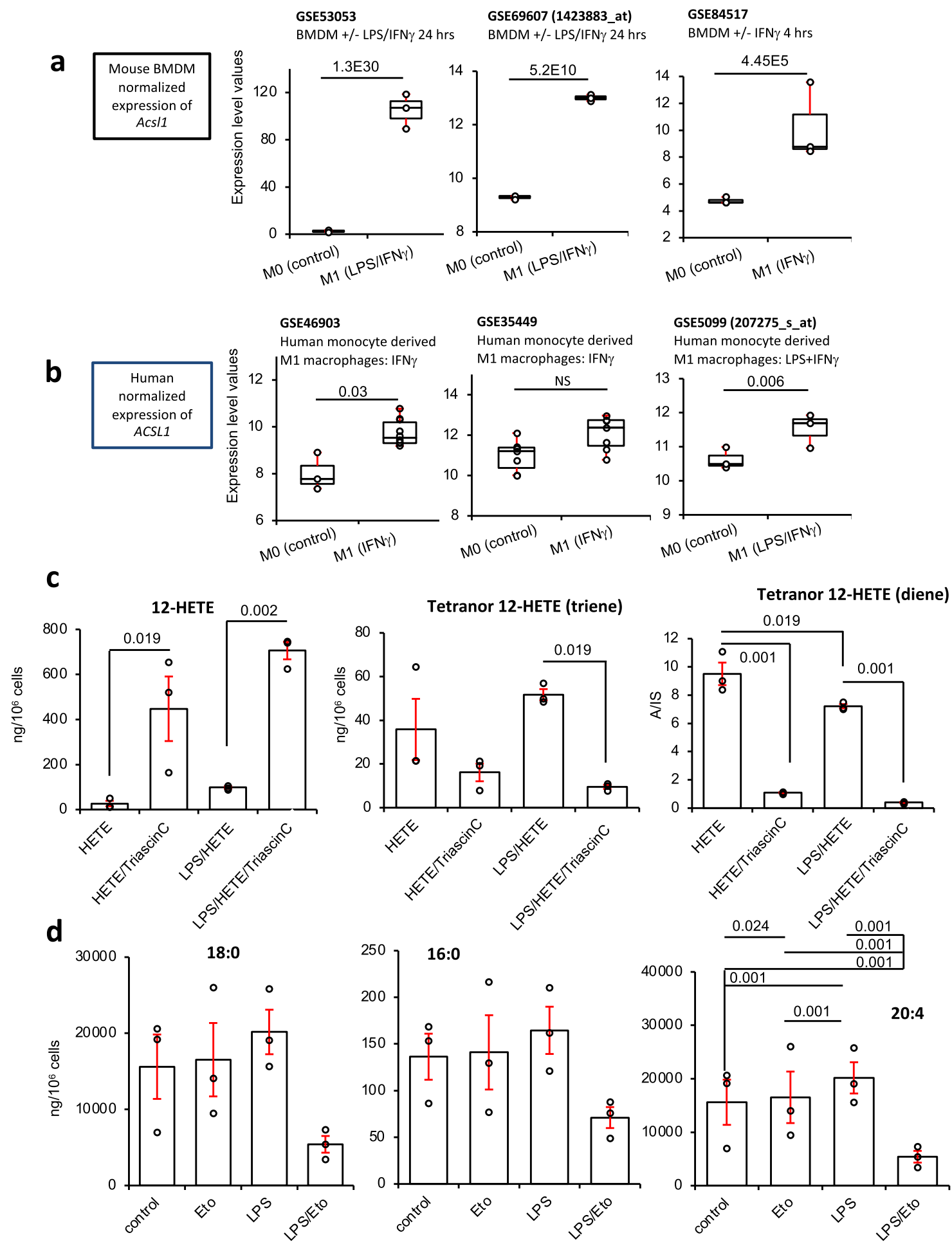

for oxylipin uptake and mitochondrial metabolism during infection (Supplementary Fig. 15). A consistent pattern across all studies was upregulation of ACSL1/Acsl1, while we also saw upregulation of ACSL3,4 (human) and Acsl5 (mice) (Figs. 5j, $6 \mathrm{a}-\mathrm{e}$, and $7 \mathrm{a}, \mathrm{b})$. Consistent with this, the pan-ACSL inhibitor, Triascin $\mathrm{C}$ was highly effective at preventing 12-HETE metabolism to the diene and triene tetranor metabolites in RAW cells (Fig. 7c). ACSL1 is already known to shuttle FAs to mitochondria for $\beta$-oxidation and lipid synthetic pathways although others may also be involved ${ }^{70-76}$. As yet, the specific ACSLs that acylate oxylipins are not known, although Klett found that all six isoforms are active in vitro ${ }^{77}$. Given our findings, characterizing which specific ACSLs support the import of oxylipins into mitochondria warrants study. ACSL1 is known to be critical for shuttling FA into mitochondria for $\beta$-oxidation in the heart, skeletal muscle, and adipose tissues ${ }^{70-74}$. Furthermore, in the liver, ACSL1 and CPT1 were shown to be physically associated $^{75,76}$. Thus, ACSL/CPT1 may be a critical checkpoint enabling higher rates of oxylipin metabolism during infection. Indeed, a critical role for ACSL1 in sepsis outcome has been proposed $^{78}$. Regulating import of long-chain FAs into the mitochondrial matrix, we found increased CPT1a or $b$, dependent on 
Fig. 7 Elevated AcsI1/ACSL1 in mouse and human GEO datasets, triascin C significantly inhibits 12-HETE removal by cells, and prevents generation of tetranor diene or triene HETE metabolites, while etomoxir alters AA levels in RAW cells. a Plots for normalized expression (using Limma and Oligo Bioconductor packages) of Acs 17 in the mouse datasets, with $n=3$ for all groups except for GSE53053 MO $(n=2)$ adjusted using Benjamini-Hochberg test. b ACSL1 is strongly induced in human M1 macrophages. Human transcriptomics data for ACSL1 expression were downloaded from GEO and normalized expression level plotted. GSE46903 ( $n=3,10$ for MO, M1 respectively), GSE35499 $(n=7)$, GSE5099 $(n=3)$. Data were normalized using Limma and Oligo Bioconductor packages as outlined in Methods, then adjusted using the Benjamini-Hochberg test. Box shows median, and interquartile ranges (IQR). The ends of the whisker are at 1.5 $\times I Q R$ above the third quartile (Q3) and $1.5 \times I Q R$ below the first quartile (Q1). If minimum or maximum values are outside this range, then they are shown as outliers. c Triascin $\mathrm{C}$ alters metabolism of 12-HETE. RAW cells were cultured for $3 \mathrm{~h}$ in serum/phenol red-free medium with 12(S)-HETE (1.4 $\mu \mathrm{g} / 10^{6}$ cells), with/without $100 \mathrm{ng} / \mathrm{ml}$ LPS with/without $7 \mu \mathrm{M}$ Triascin C. Cells and supernatant were harvested and $12-$ HETE and its tetranor metabolites measured using LC/MS/MS ( $n=3$, mean \pm SEM, separate wells of cells). Significance was tested using ANOVA with Tukey post hoc test. The impact of LPS (either with or without Triascin C) was not significant for any conditions or lipids, except for diene. $\mathbf{d}$ Etomoxir modulates levels of AA in RAW cells. RAW cells were cultured for $3 \mathrm{~h}$ in serum/phenol red-free medium with $12(\mathrm{~S})$-HETE (1.4 $\left.\mu \mathrm{g} / 10^{6} \mathrm{cells}\right)$, with/without $100 \mathrm{ng} / \mathrm{ml}$ LPS with/without $25 \mu \mathrm{M}$ etomoxir. Cells and supernatant were harvested and 16:0, 18:0, and 20:4 measured using LC/MS/MS ( $n=3$, mean \pm SEM, separate wells of cells). Significance was tested using ANOVA with Tukey post hoc test. For 16:0 and 18:0, there were no significant differences found.

the model (Fig. 6a-c), along with CPT2 induction in human sepsis (Fig. 6a). Murine BMDM stimulated with IFN- $\gamma$ also upregulated Ecil (Fig. 6d, e). This encodes DCI, which converts 3 -cis or trans-enoyl-CoA to 2-trans-enoyl-CoA during mitochondrial $\beta$-oxidation and was previously shown to be induced during Hepatitis $C$ virus infection and required for virus replication ${ }^{79,80}$. Last, upregulation of the $\beta$ subunit of the mitochondrial trifunctional protein (encoded by $H A D H B$ ) and very long-chain acyl-CoA dehydrogenase (encoded by ACADVL) are also fully consistent with their proposed role in oxylipin $\beta$ oxidation (Fig. 6a and Supplementary Fig. 15).

Macrophages rely primarily on glycolysis for energy when stimulated with LPS/IFN- $\gamma$, and conversely use FAO to supply OxPhos with substrates when exposed to immune-modulatory stimuli such as IL- $4^{26}$. However, recent studies have uncovered a significant role for lipid metabolism in "M1" cells even in the absence of OxPhos, with FA synthetic pathways (FAS) being upregulated by LPS ${ }^{26}$. Our observation that etomoxir partially suppressed 18:0 and 16:0 levels in LPS-stimulated RAW cells is consistent with this (Fig. 7d). This anabolic event is proposed to supply inflammatory activated cells with lipids for processes such as proliferation (sphingolipids, glycerolipids, phospholipids, etc.), and generation of oxylipins ${ }^{26}$. However, in the case of PUFA substrates for oxylipin synthesis, FA elongation/desaturation of the essential FA, linoleic and $\alpha$-linolenic acid by FADS and ELOVL gene products is the key event required. Although we found significant elevations in 20:4 (Fig. 7d), this was most likely mediated via $\mathrm{PLA}_{2}$ activities since there was no impact of etomoxir and FADS and ELOVL genes were not upregulated by LPS. Here, we focused down on inflammatory activated macrophages that generate oxylipins, where metabolism will favor glycolysis and FAS, and reduced OxPhos. We show for the first time that CPT1 mediates exogenous and endogenously generated oxylipin $\beta$-oxidation by macrophages, which is elevated during inflammation. The impact of blocking CPT1 varied by sub-family of oxylipins, based on either:

(i) Cell type: etomoxir consistently elevated LOX-derived oxylipins and various other monohydroxy-oxylipins, while suppressing $\mathrm{CYP} / \mathrm{sEH}$ metabolites in all macrophages. However, COX-derived PGs increased in vivo and in primary macrophages, but were suppressed in RAW cells.

(ii) How CPT1 was targeted: in RAW cells, etomoxir or gene silencing suppressed COX-derived $\mathrm{PGs}$ and $\mathrm{CYP} / \mathrm{sEH}$ oxylipins. In contrast, monohydroxy oxylipins were increased by etomoxir but suppressed by CPT1 knockdown in RAW cells. Etomoxir was used at $25 \mu \mathrm{M}$, well below concentrations found to induce off-target effects on adenine nucleotide translocase $\mathrm{e}^{36,37}$. Indeed, previous studies using high levels of etomoxir led to FAO being incorrectly proposed as required for alternative activation of $\mathrm{M} 2$ macrophages $(100-200 \mu \mathrm{M})^{81}$. Nowadays, lower concentrations are recommended that block $\sim 90 \%$ of $\beta$-oxidation without side-effects (e.g., $10-25 \mu \mathrm{M})^{38}$. As a second approach, shRNA knockdown stably reduced expression of CPT1a. However, this causes adaptive changes to the metabolic status of macrophage mitochondria, beyond acute inhibition of CPT1. Recent publications implicate CPT1 in maintaining mitochondrial health due to its requirement for importing FAs for maintaining mitochondrial cardiolipin and phospholipid pools ${ }^{38}$. Thus, cells lacking CPT1 do not proliferate normally and may have adapted to a deficiency in OxPhos through alterations in other lipid and energy metabolism pathways. Thus, constitutive knockdown may directly impact the balance between mitochondrial and peroxisomal $\beta$-oxidation and the ability to support elongase activities in the cells. Nevertheless, both inhibitory approaches caused significant changes to oxylipin secretion, both for PGs, 12-HETE and its two tetranor metabolites, supporting the hypothesis that mitochondria can be a significant site of oxylipin regulation in macrophages.

Our data reveal complex modulation of oxylipin removal and formation by CPT1 as follows:

(i) Removal: oxylipins and their metabolites can be degraded by two separate $\beta$-oxidation pathways, with only mitochondrial enzymes relying on CPT1. However, peroxisomes and mitochondria display metabolic interplay where FA degradation intermediates are transferred for metabolism between the organelles ${ }^{82}$. Thus, stable knockdown of CPT1 may require compensatory increases in peroxisomal $\beta$-oxidation. It has been proposed that truncated unsaturated FA metabolites may need to transfer to mitochondria for complete metabolism ${ }^{82}$. Indeed, 12-HETE-triene was suggested as the terminal peroxisomal metabolite for 12HETE, with further metabolism proposed to require mitochondria ${ }^{18}$. Our data provide supportive evidence for this interplay between organelles since blockade of CPT1 had a far greater impact on the metabolism of tetranor metabolites than exogenous 12-HETE in LPS-stimulated cells. Thus, 12-HETE can be metabolized in either organelle, but peroxisomal-generated tetranors are then shuttled to mitochondria, with their oxidation significantly stimulated by inflammation.

(ii) Generation: oxylipins are generated from PUFA, which are themselves synthesized via chain elongation and desaturation of linoleic and $\alpha$-linolenic acids. This process requires 
malonyl-CoA, which is formed from acetyl-CoA, a product of which themselves depend at least in part on CPT1 for formation. Thus, while both synthesis and degradation of oxylipins simultaneously occur in macrophages, steadystate levels will depend on the cells' metabolic status, and whether CPT1 is mainly supporting degradation (by $\beta$ oxidation) or synthesis (by supplying TCA intermediates) of individual oxylipins. Thus, CPT1 could either increase or decrease levels of oxylipins and their metabolites concurrently, as seen in our study. Detailed flux analysis of this phenomenon is required to further our understanding since a role for regulation of elongation/desaturation was not clearly seen in our study.

Here, we show that mitochondrial oxylipin removal takes place during inflammation on a background of reduced OxPhos, a wellknown response to LPS stimulation by macrophages ${ }^{28}$. However, $\beta$-oxidation requires a basal level of OxPhos both to regenerate $\mathrm{NAD}+$ and oxidized flavin cofactors in the electron transferring flavoprotein complex, which reduces ubiquinone. While OxPhos was suppressed, there was a low residual activity remaining in both RAW and peritoneal cells that appeared sufficient to sustain $\beta$-oxidation, observed by the formation/metabolism of oxylipins and HETE tetranors. Our experiments using exogenous 12-HETE suggest that non-mitochondrial pathways such as peroxisomal oxidation and also esterification is probably responsible for removing most. This agrees with previous studies where exogenous 12-HETE added to mammalian cells was removed by peroxisomes forming a series of metabolites, down to C12:1 18,34,83,84, and a significant amount is converted to esterified pools ${ }^{35}$. However, these studies did not consider the impact of inflammation and the focus was exclusively on exogenous 12 HETE, not endogenous oxylipins generated by the cells. Here, we extend this range of oxylipins generated by macrophages, and reveal that during LPS inflammation, mitochondria (via CPT1) effectively halve secreted amounts of oxylipins en masse, and are a key site for removing peroxisomal metabolites of 12-HETE. We demonstrate that this alters the outcome of leukocyte activation, suggesting that mitochondrial $\beta$-oxidation may act as a brake for dampening host inflammatory responses in vivo during infection. Further studies are required to test this idea in vivo, including characterization of the ability of other leukocytes such as neutrophils and $\mathrm{T}$ cells to remove oxylipins via $\beta$-oxidation The potential implications for humans with CPTla deficiency, relating to severely augmented inflammation during infection should be considered and studies initiated to test this idea. Also, whether this phenomenon contributes to infection-associated host inflammation in general, in cases where mitochondrial health is compromised, should be considered.

\footnotetext{
Methods

Mouse peritonitis model. All mouse experiments were performed in accordance with the United Kingdom Home Office Animals (Scientific Procedures) Act of 1986 (P05K6A456) and for peritoneal models under (UK Home Office PPL 30/ 2938, and PPL P05D6A456). Wild-type female C57BL/6 (strain code 632) mice were purchased for all studies from Charles River Laboratories. Mouse breeders were housed in isolators (barrier) and all experimental mice in individually ven tilated cages with 12 -h light/dark cycles and controlled temperature $\left(20-22^{\circ} \mathrm{C}\right)$ (specific pathogen-free/SPF). Access to water and standard chow was ad libitum, and humidity was atmospheric, and not specifically regulated. Female C57BL/6 mice wild-type mice (7-9 weeks) were injected intraperitoneally with etomoxir (100 $\mu$ g, Sigma-Aldrich, Cat. No E1905) and/or LPS (1 $\mu$ g, E. coli 0111.B4, SigmaAldrich, Cat. No L3024) or vehicle (PBS) per mouse. After $6 \mathrm{~h}$, mice were euthanized using $\mathrm{CO}_{2}$, then peritoneally lavaged using $5 \mathrm{ml}$ ice-cold PBS (with $5 \mathrm{mM}$ EDTA). Eicosanoid internal standard mix was added to the lavage solution $(7.5 \mu \mathrm{l}$ per $5 \mathrm{ml}$ lavage solution, Supplementary Data File 2). Cells were separated by centrifugation and resuspended in $1 \mathrm{ml}$ PBS. Supernatant and cells were frozen at $-80^{\circ} \mathrm{C}$ until lipid extraction using solid phase as described below.
}

Isolation and treatment of resident peritoneal macrophages. Wild-type mice (female, 9-13 weeks) were lavaged with PBS supplemented with 5 mM EDTA, and peritoneal cells from four to five mice pooled together. A small amount was labeled with F4/80 (BD Horizon, BV421 Rat Anti-Mouse F4/80, Clone T45-2342, Cat.: 565411) and CD11b-allophycocyanin antibodies (BD Pharmingen, Clone M1/70, PE Rat Anti-Mouse, Cat 557397), both $0.2 \mu \mathrm{g} / \mathrm{ml}$ final concentration, to determine macrophage numbers. Cells were first blocked for non-specific antigen binding (incubating for $20 \mathrm{~min}$ at RT with $2 \mu \mathrm{g} / \mathrm{ml} \mathrm{CD16/CD} 32$ Rat anti-Mouse, unlabeled, Clone: $2.4 \mathrm{G} 2, \mathrm{BD}, 553142$ ) then antibodies were added without washing off the blocking solution, and cells were incubated for further $20 \mathrm{~min}$ at RT. Cells were then centrifuged for $5 \mathrm{~min}$ at $350 \mathrm{~g}, 4^{\circ} \mathrm{C}$, supernatant discarded and cells resuspended in $150 \mu \mathrm{l}$ PBS and \% macrophages determined using flow cytometry. Peritoneal cells were seeded at $10^{6}$ macrophages/well of a 6-well plate and cultured in DMEM (with $10 \%$ heat-inactivated FCS and pen/strep), for $2 \mathrm{~h}$ at $37^{\circ} \mathrm{C}, 5 \%$ $\mathrm{CO}_{2}, 99 \%$ humidity. Nonadherent cells were removed by washing with media, and the remaining cells cultured in serum-free, phenol red-free, RPMI, with/without LPS $(100 \mathrm{ng} / \mathrm{ml})$ and/or etomoxir $(25 \mu \mathrm{M})$. After $24 \mathrm{~h}$, cells and supernatants were harvested separately, with supernatant first centrifuged $\left(350 \mathrm{~g}, 5 \mathrm{~min}, 4^{\circ} \mathrm{C}\right)$, and cells scraped quickly into $1 \mathrm{ml}$ RPMI. Supernatant and cells were then snap-frozen in liquid nitrogen and stored at $-80^{\circ} \mathrm{C}$, prior to lipid extraction and analysis. For analysis of TNF and CCL5/RANTES, supernatants were analyzed using commercial ELISA assay kits (R \& D Systems; Mouse TNF DuoSet ELISA, Cat. No DY41005; Mouse CCL5/RANTES DuoSet ELISA, Cat. No DY478-05) according to the manufacturer's protocol.

\section{Generation and culture of RAW264.7 cell lines stably overexpressing Alox15} or expressing shRNA targeting Cpt1a. Alox15 overexpressing RAW264.7 cells were generated by retroviral transduction using the PMX-IP retroviral vector, which is based on the Moloney Murine Leukemia Virus (MMLV) ${ }^{85}$. Briefly, mRNA was extracted from C57BL/6 peritoneal cells, and Alox15 was PCR amplified with the following primer sequences:

- Alox15-Fw: TCCTGCAGGCCTCGAGCCACCATGGGTGTCTACCGCAT CC

\section{- Alox15-Rv: CGCGCCGGCCCTCGAGTCATATGGCCACGCTGTTTT}

The In-fusion cloning kit (Takara Bio, 102518) was utilized to insert Alox15 into the Xho I site of the pMXs MMLV retroviral plasmid. Retroviral particles were generated after transfection of HEK293T cells with the viral plasmid. RAW264.7 cells were spin-infected with packaged retrovirus (Alox15 or empty vector control pMXs-IP). Positive infections were selected by resistance to $3 \mu \mathrm{M}$ puromycin, screened for positive Alox15 expression by PCR and 12-HETE generation using LC/MS/MS (Supplementary Fig. 11a). Separately, RAW264.7 cells were infected with lentiviral vectors to generate Cpt1a knockdown or non-silencing control cell lines, as described ${ }^{86}$. Alterations from the published method were different lentiviral plasmids (pSMART mEFla/TurboGFP (Cptla targeting shRNA [V3SVMM03-13316872] and non-targeting control)-Dharmacon). Briefly, lentiviral particles were created by transfecting HEK293T cells (lentiviral plasmid plus helper constructs (pCMV- $88.91(\mathrm{Gag} / \mathrm{Pol}$, Tat and Rev) and pMD2.G (VSV$\mathrm{G}$ coat)) using the Effectene transfection reagent (Qiagen). Lentivirus was concentrated $48 \mathrm{~h}$ after transfection from $0.45 \mu \mathrm{m}$ filtered supernatants layered on top of 1/6th volume of $0.584 \mathrm{M}$ sucrose using a Beckman Coulter Ultracentrifuge $\left(100,000 \times \mathrm{g}, 90 \mathrm{~min}, 4^{\circ} \mathrm{C}\right)$. Lentiviral particles concentrated from $20 \mathrm{ml}$ of supernatant were added to a $~ 10 \%$ confluent RAW264.7 cells in a T-175 flask for 3 days. After another 4 days of subsequent cell expansion, positively infected cells were sorted using GFP expression on a BD FACS Aria cell sorter $(100 \mu \mathrm{m}$ nozzle size) (Supplementary Fig. 9c), using Attune NxT software (V2). Knockdown was validated by real-time PCR. Mature Cpt1a mRNA target sequence: CAGTGGTA TTTGAAGCTAA, the sequence of the non-targeting control was not disclosed by Dharmacon but is validated to have minimal targeting to known mouse genes. To confirm knockdown, RNA was extracted using the PureLink RNA extraction kit, cDNA was created using the high capacity reverse transcription kit (Life Technologies). Real-time PCR was performed using the Taqman assay (Applied Biosystems) on a Stratagene MX3000P real-time PCR instrument. Data were analyzed using the $\Delta \Delta \mathrm{Ct}$ method, normalizing Cptla (probe spans exons 13-14: Mm01231183_m1) gene expression to the housekeeping genes Hprt (probe spans exons 2-3: Mm03024075_m1) and Actb (probe spans boundary of exon 3: Mm02619580_g1) (Supplementary Fig. 9a). RAW cells were cultured in DMEM with $10 \%$ FCS and pen/strep. Cells were passaged at $~ 80 \%$ confluency using PBS with $5 \mathrm{mM}$ EDTA and $27.7 \mathrm{mM}$ lidocaine for $\sim 5 \mathrm{~min}$ at $37^{\circ} \mathrm{C}$. Cells were cultured with/without $100 \mathrm{ng} / \mathrm{ml} \mathrm{LPS}$, with/without $25 \mu \mathrm{M}$ etomoxir for $24 \mathrm{~h}$ in serum-free RPMI medium. Then cells and supernatants were harvested separately and stored at $-80^{\circ} \mathrm{C}$ until lipid extraction and analysis using LC/MS/MS

Bone marrow-derived macrophages (BMDM) isolation and differentiation. Bone marrow cells were harvested from femurs and tibias of mice (8-10 weeks old, female C57BL/6) using syringe flushing. After centrifugation and washing twice with PBS cells were plated at $2 \times 10^{5} / \mathrm{ml}$ in DMEM ( $10 \%$ FCS, $\pm 5 \%$ horse serum, pen/strep, L-glutamine, \pm HEPES). MCSF was added at a final concentration $20 \mathrm{ng} /$ ml. On day 3, cells were refed using media and MCSF. On day 7-8, cells were 
washed with PBS, then lifted with lidocaine/EDTA or ACCUTASE ${ }^{\mathrm{mt}}$ (SigmaAldrich), plated at $1-2 \times 10^{6}$ per well of a 6-well plate and rested for $24 \mathrm{~h}$. Cells were washed and then refed with DMEM (as above, with MCSF) and cytokines as follows: M0: medium only, M1: LPS (100 ng/ml)/IFN- $\gamma(20 \mathrm{ng} / \mathrm{ml})$, M2: IL-4 $(20 \mathrm{ng} / \mathrm{ml})$. For some experiments, FCS was omitted. Etomoxir $(25 \mu \mathrm{M})$ was added in some experiments. After $24 \mathrm{~h}$ supernatant was recovered and frozen at $-80^{\circ} \mathrm{C}$. Cells were washed and then recovered in $1 \mathrm{ml}$ of PBS using rapid scraping on ice, and the cell suspension was immediately quenched in liquid $\mathrm{N}_{2}$ prior to storage at $-80^{\circ} \mathrm{C}$.

Samples were analyzed for phenotype confirmation (cytokine production, mRNA gene expression by real-time PCR) and lipid composition, as described. CCL5, TNF, and IL-6 in supernatants were measured using ELISAs (Mouse TNF; Mouse CCL5; Mouse IL-6, R\&D Systems) according to the manufacturer's protocols. Confirmation of phenotype is shown in Supplementary Fig. 11b, c. Total RNA was recovered using the RNeasy Mini Kit (QIAGEN, 74104) according to the manufacturer's instructions. RNA concentration and purity were determined using a Nanodrop spectrophotometer. cDNA was prepared from $1 \mu \mathrm{g}$ of RNA. RNA was reverse-transcribed using superscript III reverse transcriptase (Invitrogen) in a total volume of $20 \mu \mathrm{L}$ for $1 \mathrm{~h}$ at $50^{\circ} \mathrm{C}$, and the reaction was terminated at $70^{\circ} \mathrm{C}$ for 15 min. Power SYBR Green PCR Master Mix (Thermo Fisher Scientific) was used for real-time PCR. The Master Mix (10 $\mu \mathrm{l})$ was added to $\mathrm{dH} 2 \mathrm{O}(7.5 \mu \mathrm{l})$, and $1 \mu \mathrm{l}$ primer mix $(0.5 \mu \mathrm{g} / \mathrm{ml}$ each primer) to make a gene-specific master mix; cDNA $(1.5 \mu \mathrm{l})$ from each sample was added to the reaction mix $(17.5 \mu \mathrm{l})$ in a 96 -well plate well. The plate was inserted into a QuantStudio 3 Real-Time PCR system and the PCR amplification started as per instrument instruction: $50^{\circ} \mathrm{C} 2 \mathrm{~min} ; 95^{\circ} \mathrm{C} 10 \mathrm{~min}$; $95^{\circ} \mathrm{C} 15 \mathrm{~s} ; 60^{\circ} \mathrm{C} 1 \mathrm{~min}$; Repeat $3-4$ for 40 cycles; $95^{\circ} \mathrm{C} 15 \mathrm{~s} ; 4^{\circ} \mathrm{C}$ end. Primers used for real-time PCR were designed to be intron-spanning, and to have a melting temperature $55-65^{\circ} \mathrm{C}$. The intron-spanning aspect ensured that only mRNA and not genomic DNA (gDNA) were amplified. All primers and oligonucleotides were obtained (Sigma, Gillingham, UK) as salt-free purified lyophilized DNA. The sequences of the real-time PCR primers used are shown in Supplementary Table 3.

Metabolism of exogenous 12-HETE and 12-HETE-d8 by RAW cells. RAW cells $\left(2 \times 10^{6} / \mathrm{ml}\right.$ media) were supplemented with $9 \mu \mathrm{M} 12$ (S)-HETE, 12 (S)-HETE-d8, 12(R)-HETE tetranor 12(S)-HETE (from Cayman Chemical) for up to $12 \mathrm{~h}$ in serum-free RPMI, with $100 \mathrm{ng} / \mathrm{ml}$ LPS, with/without $25 \mu \mathrm{M}$ etomoxir. Amounts supplemented were (per $10^{6}$ cells): $12 \mathrm{~h}$ timecourse $12(\mathrm{~S}$ )-HETE: $1.44 \mu \mathrm{g} .3 \mathrm{~h}$ fixed time supplementation experiments: 12(S)-HETE/12(R)-HETE, 12(S)-HETE-d8: $1.5 \mu \mathrm{g}$, tetranor 12(S)-HETE triene: $1.2 \mu \mathrm{g}$. Following incubation, the supernatant was harvested, centrifuged to remove any dead/dying cells and then snap-frozen. Cells were gently rinsed with PBS to remove any dead/dying cells. Following this, the cells were scraped in PBS and snap-frozen. Samples were stored at $-80^{\circ} \mathrm{C}$. Lipids were extracted as described below, using solid-phase $\mathrm{C} 18$ columns, resuspended in small volumes of methanol and stored at $-80^{\circ} \mathrm{C}$ until analysis using LC/ MS/MS. For experiments testing the impact of Triascin C, cells and supernatant were together scraped and harvested before snap freezing, then processed as outlined below for lipid extraction and analysis.

Lipid extraction. Internal standard mixture (see eicosanoid assay for details, Supplementary Data File 2) was added to each sample before extraction. Oxylipins were extracted from cells by adding $2.5 \mathrm{ml}$ of solvent mixture $(1 \mathrm{M}$ acetic acid/2isopropanol/hexane $(2: 20: 30, \mathrm{v} / \mathrm{v} / \mathrm{v})$ to $1 \mathrm{ml}$ of sample, followed by vortexing for $60 \mathrm{~s}$, and then adding $2.5 \mathrm{ml}$ of hexane. The mixture was vortexed again for $60 \mathrm{~s}$, centrifuged at $500 \mathrm{~g}, 4^{\circ} \mathrm{C}$ for $5 \mathrm{~min}$, and lipids recovered in the upper organic solvent layer. The lipids were reextracted by adding another $2.5 \mathrm{ml}$ of hexane, followed again by vortexing and centrifugation. The lipids were again recovered in the upper organic solvent layer. The combined hexane layers were dried by vacuum and resuspended in methanol. Oxylipins were extracted from supernatants using solid-phase extraction (SPE) using C18 columns (Sep-Pak C18, Waters). Columns were activated using $12 \mathrm{ml}$ methanol, followed by $6 \mathrm{ml}$ acidified water $(0.4 \%$ glacial acetic acid in HPLC water) followed by loading the sample (all samples were in $85 \%$ aqueous, $15 \%$ methanol, $\mathrm{pH} \mathrm{3.0)}$ ), and washing first with $10 \mathrm{ml}$ acidified water ( $0.4 \%$ glacial acetic acid in HPLC water), then with $6 \mathrm{ml}$ of hexane. The columns were left to dry for $15-20 \mathrm{~min}$ followed by eluting the lipids with $8 \mathrm{ml}$ methyl formate. The lipids were vacuum dried, resuspended in methanol and stored at $-80^{\circ} \mathrm{C}$.

LC-MS/MS targeted analysis of oxylipins. Targeted lipidomics analysis of 93 oxylipins was performed on a Nexera liquid chromatography system (Nexera X2, Shimadzu) coupled to a 6500 QTrap mass spectrometer (AB Sciex) as described, with minor adaptations $s^{87}$. Briefly, liquid chromatography was performed at $45^{\circ} \mathrm{C}$ using a Zorbax Eclipse Plus C18 (Agilent Technologies) reversed-phase column $(150 \times 2.1 \mathrm{~mm}, 1.8 \mu \mathrm{m})$ at a flow rate of $0.5 \mathrm{ml} / \mathrm{min}$ over $22.5 \mathrm{~min}$. Mobile phase A was (95\% HPLC water/5\% mobile phase B; $\mathrm{v} / \mathrm{v}$ and $0.1 \%$ acetic acid) and mobile phase $\mathrm{B}$ was acetonitrile/methanol $(800 \mathrm{ml}+150 \mathrm{ml}$; and $0.1 \%$ acetic acid). The following linear gradient for mobile phase B was applied: $30 \%$ for $1 \mathrm{~min}, 30-35 \%$ from 1 to $4 \mathrm{~min}, 35-67.5 \%$ from 4 to $12.5 \mathrm{~min}, 67.5-100 \%$ from 12.5 to $17.5 \mathrm{~min}$ and held at $100 \%$ for $3.5 \mathrm{~min}$, followed by $1.5 \mathrm{~min}$ at the initial condition for column re-equilibration. The injection volume was $5 \mu \mathrm{L}$. Lipids were analyzed in scheduled multiple reaction monitoring mode with a detection window of $55 \mathrm{~s}$ for each analyte. Ionization was performed using electrospray ionization in the negative ion mode with the following MS parameters: temperature $475^{\circ} \mathrm{C}, \mathrm{N}_{2}$ gas, GS1 $60 \mathrm{psi}$, GS2 $60 \mathrm{psi}$, curtain gas $35 \mathrm{psi}$, ESI voltage $-4.5 \mathrm{kV}$. Cycle time was $0.4 \mathrm{~s}$. Peak areas for lipids were integrated and normalized for deuterated internal standards and quantification was performed using external calibration curves with authentic standards for all investigated oxylipins. Data were acquired using Analyst (V1.6) integration and quantification was performed using MultiQuant software (version 3.0.2, AB Sciex Framingham, MA, USA). A full list of the internal standards and oxylipins is provided in Supplementary Data File 2 along with calibration range, MRM transitions and corresponding MS parameters (collision energy, declustering potential). See Supplementary Data File 1 for a list of abbreviations and formal names for lipids measured. In this assay, identification is based on retention time comparison with authentic standards and the presence of a diagnostic precursor and product ion pair; however, stereochemistry is not established and in the case of lower abundance lipids, is often unclear. For that reason, chirality has been removed. LOQ for each lipid was set at signal:noise 5:1, and the primary standard amount (ng on column) closest to this value for each lipid is shown in Supplementary Data File 2, column N. All chromatograms from tissue/cell samples were manually checked for peak quality and excluded from analysis if they fell below the limit of 5:1 (signal:noise, LOQ) with a minimum of six points across each peak. Where lipids fell below LOQ, a zero value was recorded, but replaced with $50 \% \mathrm{LOQ}$ for statistical analysis. Example chromatograms for all lipids analyzed are provided in Supplementary Fig. 12.

Targeted lipidomics analysis of 12(S)-HETE, 12(R)-HETE, 12(S)-HETE-d8, or tetranor 12(S)-HETE metabolites was performed on a Nexera LC coupled to a 4000 QTrap (AB Sciex). Briefly, liquid chromatography was performed at $40^{\circ} \mathrm{C}$ using a Spherisorb ODS2 C18 column $(4.6 \times 150 \mathrm{~mm}, 5 \mu \mathrm{m}$, Waters $)$ at a flow rate of $1 \mathrm{ml} /$ min over $30 \mathrm{~min}$. Mobile phase A was (75\% HPLC water/25\% acetonitrile; $\mathrm{v} / \mathrm{v}$ and $0.1 \%$ acetic acid) and mobile phase B was $60 \%$ methanol $/ 40 \%$ acetonitrile; $\mathrm{v} / \mathrm{v}$ and $0.1 \%$ acetic acid. The following gradient for mobile phase B was applied: $50-90 \%$ B over $20 \mathrm{~min}$, then held at $90 \%$ B for $5 \mathrm{~min}$, followed by re-equilibration to $50 \%$ B from 25 to $30 \mathrm{~min}$. The injection volume was $10 \mu$ l. Ionization was performed using electrospray ionization in the negative ion mode with the following MS parameters: temperature $650^{\circ} \mathrm{C}, \mathrm{N}_{2}$ gas, GS1 $60 \mathrm{psi}$, GS2 $30 \mathrm{psi}$, curtain gas $35 \mathrm{psi}$, ESI voltage $-4.5 \mathrm{kV}$. Cycle time was $1.46 \mathrm{~s}$. Peak areas for lipids were integrated and quantification was performed using an external calibration curve with 15-HETE-d8 as standard for all investigated compounds. As no primary standard is available for the tetranor diene, data are expressed as analyte/internal standard signal ratio (A/ IS). Data integration and quantification was performed using MultiQuant software (version 3.0.2, AB Sciex Framingham, MA, USA). MRM transitions and corresponding MS parameters (collision energy and declustering potential) for each analyzed lipid are provided in Supplementary Table 4.

Mitochondrial stress test. Oxygen consumption rate (OCR) was measured using Seahorse XFe96 analyzer (Agilent, with Wave 2.3/4 software) and the Seahorse XFe96 FluxPak (102416-100, Agilent) according to the manufacturer's protocols. Briefly, RAW/RAWAlox 15 cells were seeded at $2.5 \times 10^{5}$ cells/well and peritoneal macrophages were seeded at $5 \times 10^{5}$ cells/well (and incubated with LPS (100 ng/ $\mathrm{ml})$ ). Mitochondrial function was assessed using inhibitors (all from Sigma) injected in the following order: oligomycin $(1 \mu \mathrm{M})$, FCCP (Carbonyl cyanide 4(trifluoromethoxy)phenylhydrazone, 2 or $4 \mu \mathrm{M}$ final concentration for peritoneal macrophages or RAW cells, respectively), rotenone $(1 \mu \mathrm{M})$, antimycin A $(10 \mu \mathrm{M}$ for peritoneal macrophages; $1 \mu \mathrm{M}$ for RAW/RAWAlox15 cells). OCR is described as $\mathrm{pmol} / \mathrm{min}$ (per well).

Heatmap method. For the generation of heatmaps, amounts (ng/ $10^{6}$ cells) of each lipid were averaged, then $\log 10$ was applied to the mean value. Heatmaps were generated using Pheatmap (V1) in R (3.6.3). The Euclidean metric was chosen to establish the treatments' relationships depicted as clusters ${ }^{88}$. The clusters are aggregated following the "shortest distance" rule. Lipids are color coded by putative enzymatic origin (Supplementary Data File 1).

\section{Determination of reactive oxygen species generation by peripheral blood}

cells. Human blood from healthy workplace volunteers was obtained with ethical approval from School of Medicine (SMREC 16/02) with informed consent. Volunteers were male or female, healthy, between the ages of 25 and 55, with no genotype information. There was no participant compensation. Whole blood $(5 \mathrm{ml})$ was drawn using a butterfly syringe into $50 \mu \mathrm{l}$ heparin sodium; $12 \mu \mathrm{l}$ of this was then placed in a microcentrifuge tube and $1 \mathrm{ml}$ cold PBS added. Following centrifugation $\left(350 \times \mathrm{g}, 5 \mathrm{~min}, 4^{\circ} \mathrm{C}\right)$ the cell pellet was recovered and resuspended, then stained with anti-CD15 BV510 (1:50 in $0.5 \mathrm{ml}$ PBS, W6D3, Becton Dickinson, $563141)$ for $30 \mathrm{~min}$ on ice. Cells were centrifuged $\left(350 \times \mathrm{g}, 5 \mathrm{~min}, 4^{\circ} \mathrm{C}\right)$ and resuspended in DMEM media ( $2 \mathrm{mM}$ glutamine) containing APF (1:1000, cell concentration $=$ cells from $5 \mu \mathrm{l}$ blood per $\mathrm{ml}$ of APF media). S. epidermidis bacteria (clinical isolate) ${ }^{56}$ were killed by incubation at $65^{\circ} \mathrm{C}$ for $20 \mathrm{~min}$, then washed by centrifugation and resuspended in PBS three times $(12,000 \times \mathrm{g}, 5 \mathrm{~min})$. Bacteria were counted by flow cytometry using an Attune NxT; bacteria were excluded from other particles by size (Forward-scatter vs Side-scatter). Bacteria were first added to blood cells at varying doses, to determine optimal reactivity per donor isolate 
(ranging from $1.37 \times 10^{5}$ to $2.75 \times 10^{6}$ bacteria per $\mathrm{ml}$ of APF media containing cells from $5 \mu \mathrm{l}$ blood). For this, cells + bacteria were incubated for $20 \mathrm{~min}$ at $37^{\circ} \mathrm{C}$, then tubes were placed on ice. Cells were washed three times with ACK red blood cell lysing buffer $\left(350 \times \mathrm{g}, 5 \mathrm{~min}, 4^{\circ} \mathrm{C}\right)$ then analyzed by flow cytometry. Cell gating strategies are shown in Supplementary Fig. 11d-f. This included forward- vs sidescatter profile (FSC vs SSC) that distinguishes neutrophils and monocytes from lymphocytes. Neutrophils were further separated from eosinophils and monocytes via their high expression of CD15. The bacterial dose was selected based on a neutrophil $\mathrm{APF}^{+}$response of between 10 and $40 \% \mathrm{APF}^{+v e}$ cells. Once this was optimized, the experiment was repeated in triplicate for each donor, and using three individual donors, using a fresh aliquot of blood. The additional step following APF addition was that lipids or ethanol vehicle (1:100) were added and samples incubated at $37^{\circ} \mathrm{C}$ for 10 min before the bacteria addition step. Lipids used and final concentrations are provided in Supplementary Table 1. Calculations assumed a $100 \mu \mathrm{l}$ peritoneal volume in vivo (Supplementary Table 1$)^{89}$. Next, bacteria were added, cells were incubated for $20 \mathrm{~min}$ at $37^{\circ} \mathrm{C}$, then tubes placed on ice, washed, and analyzed using flow cytometry for $\mathrm{APF}^{+\mathrm{ve}}$ neutrophils or monocytes. Means for each donor were calculated separately and compared using two-way ANOVA with Sidak's post test, where $p<0.05$ was considered significant.

T-cell activation assay. All procedures were performed at $4{ }^{\circ} \mathrm{C}$, centrifugation steps were $350 \times \mathrm{g}$ for $5 \mathrm{~min}$, and reagents and equipment were from Thermo Fisher and antibodies from BioLegend unless stated otherwise. Whole blood was acquired with heparin sodium (Sigma-20 USP units per $\mathrm{ml}$ ) and gently layered onto 2/3 volume of Histopaque 1077 in a centrifuge tube. Blood was centrifuged at room temperature at $400 \times \mathrm{g}$ for $15 \mathrm{~min}$ (low acceleration and deceleration), the PBMC layer and half the serum and histopaque volume were removed to a separate tube. Six times volume of cold PBS was added, and cells were centrifuged. The supernatant was removed completely, and cells were resuspended in serum-free RPMI media (+L-glutamine) for counting, RPMI was added to achieve 5 million per $\mathrm{ml}$ (centrifugation and resuspension required-acts as an additional wash). PBMCs (50 $\mu$ l, 250,000 cells) were added to separate wells of a 96-well U-bottom plate and incubated at $37^{\circ} \mathrm{C}$ for $20 \mathrm{~min}$. Lipid mixes $(25 \mu$ l of $4 \times$ concentrated high, low or ethanol control in media) were added and incubated for $15 \mathrm{~min}$ at $37^{\circ} \mathrm{C}$. Anti-CD3/CD28 Dynabeads $(25 \mu \mathrm{l}$ of $4 \times$ concentrated in media $=0.64$ beads per PBMC) or control were then added and incubated for $2 \mathrm{~h}$ at $37^{\circ} \mathrm{C}$. Golgi Plug and Golgi Stop (BD biosciences, $5 \mu \mathrm{l}$ of $20 \times$ concentrated in media) were added for the last $4 \mathrm{~h}$ of stimulation at $37^{\circ} \mathrm{C}(6 \mathrm{~h}$ total). Ice-cold wash solution (PBS $+5 \mathrm{mM}$ EDTA $+5 \%$ BSA) was added to each well and cells were mixed and transferred to a 96-well v-bottom plate. The plate was centrifuged, and the supernatant removed. Cells were resuspended in $25 \mu$ l block solution (wash plus 1 in 40 Human TruStain FcX, BioLegend) and incubated for $5 \mathrm{~min}$. Surface stain antibody cocktail was added-25 $\mu \mathrm{l}$ wash containing $0.5 \mu \mathrm{l}$ Zombie Live/Dead Aqua, $0.25 \mu \mathrm{l}$ anti-CD8 BV-711 (RPA-T8) (BioLegend, cat \# 301044, used at 1:100 dilution), $1 \mu \mathrm{l}$ anti-CD4 BV605 (OKT4) (BioLegend, cat \# 317438, used at 1:25 dilution), $0.5 \mu \mathrm{l}$ anti-CD3 Percp (SK7) (BioLegend, cat \# 344814, used at 1:50 dilution). The plate was incubated at room temperature for $20 \mathrm{~min}$. Wash solution $(150 \mu \mathrm{l})$ was added and cells centrifuged, the supernatant was removed and cells were washed again with wash solution. Cells were resuspended in $100 \mu \mathrm{l}$ Fix/Perm solution (BD Biosciences) and incubated for $30 \mathrm{~min}$. Wash solution $(100 \mu \mathrm{l})$ was added and cells centrifuged, the supernatant was removed, and cells were washed again with wash solution. Wash solution $(150 \mu \mathrm{l})$ was added, and cells were stored overnight. The plate was centrifuged, and the supernatant removed, cells were resuspended in internal antibody mix $-50 \mu \mathrm{l}$ perm-wash (BD biosciences) with $1 \mu \mathrm{l}$ anti-TNF APC-Vio770 (cA2) (Miltenyi Biotec, cat \# 130-120-169, used at 1:50 dilution). The plate was incubated for $30 \mathrm{~min}$. Perm-wash solution $(150 \mu \mathrm{l})$ was added and cells centrifuged, the supernatant was removed and cells were washed again twice with the perm-wash solution. Cells were resuspended in $0.5 \mathrm{ml}$ wash solution and acquired on a flow cytometer. Anti-mouse IgG- $\kappa$ compensation particles were used for single stain compensation controls (BD Biosciences, cat \# 552843, 1 drop of beads per $100 \mu \mathrm{l}$ staining buffer. 50ul used per compensation control tube). Live, single cells were gated, and debris was removed before analysis of $\mathrm{CD} 3$ positive events.

Assessment of mitochondrial DNA in LPS-treated cells. RAW cells were seeded in 6-well plates at $1 \times 10^{6}$ cells/well and grown in DMEM with $10 \%$ FCS, $2 \mathrm{mM}$ glutamine and $100 \mathrm{U} / \mathrm{ml}$ of penicillin/streptomycin for $24 \mathrm{~h}$. Next, the medium was aspirated, and LPS $(100 \mathrm{ng} / \mathrm{ml})$ or chloroquine $(40 \mathrm{nM})$ was added, and cells were incubated for $24 \mathrm{~h}$. Total DNA was extracted from cells with PureLink Genomic DNA kit (Fisher Scientific, cat. No. 10053293) according to the manufacturer's instructions and quantified using a Nanodrop (Thermo Scientific). Assessment of mtDNA copy numbers, relative to gDNA, was done as previously described ${ }^{90}$. Briefly, quantitative PCR (qPCR) analysis was performed using PerfeCta ${ }^{\mathrm{TM}}$ SYBR Green FastMix ${ }^{\mathrm{TM}}$, low ROX ${ }^{\mathrm{TM}}$ (Quanta Biosciences) and primers specific for mouse mtDNA (mouse mtDNA forward: 5'-CCCCAGCCATAACACAGTATCA AAC- $3^{\prime}$, mouse mtDNA reverse: $5^{\prime}$-GCCCAAAGAATCAGAACAGATGC-3') or the genomic $18 \mathrm{~S}$ ribosome gene (mouse $18 \mathrm{~S}$ forward: $5^{\prime}$-AAACGGCTACCACATCCAAG- ${ }^{\prime}$, mouse $18 \mathrm{~S}$ reverse: CAATTACAGGGCCTCGAAAG- ${ }^{\prime}$ ) (Merck $\mathrm{KGaA}$ ). Primers specific for $\mathrm{mtDNA}$ gives rise to a $201 \mathrm{bp}$ product, while primers specific for the $18 \mathrm{~S}$ ribosomal gene gives a product that is $112 \mathrm{bp}$. qPCR was performed in a Stratagene MX3000P machine (Stratagene California, San Diego, CA, USA) using 96-well Non-Skirted, White PCR Plates (ABgene, UK) and MicroAmp Optical Caps (Applied Biosystems, UK). Each reaction was performed on $25 \mathrm{ng}$ diluted total DNA in a volume of $20 \mu \mathrm{l}$, under the following conditions: $95^{\circ} \mathrm{C}$ for $2 \mathrm{~min} ; 95^{\circ} \mathrm{C}$ for $15 \mathrm{~s}, 60^{\circ} \mathrm{C}$ for $1 \mathrm{~min}$, repeated 40 cycles; $95^{\circ} \mathrm{C}$ for $1 \mathrm{~min}$ $55^{\circ} \mathrm{C}$ for $30 \mathrm{~s}, 95^{\circ} \mathrm{C}$ for $30 \mathrm{~s}$. mtDNA/gDNA was generated by normalizing the quantified mtDNA values to the quantified 18S DNA values. To calculate mtDNA damage based on lesion frequency, the DNA of the cells was isolated as described above and assessment of mtDNA damage was performed using qPCR as previously described ${ }^{90}$. A common reverse primer (mouse mtDNA reverse: $5^{\prime}$-GCCCAAAGAATCAGAACAGATGC- $3^{\prime}$ ) in combination with primers called either forward short PCR primer (5'-GCAAATCCATATTCATCCTTCTCAAC-3') or forward long PCR primer (5'-CCCAGCTACTACCATCATTCAAGTAG-3'). The reverse primer with the short forward primer gives rise to a $16.2 \mathrm{~kb}$ product, or with the long forward primer gives rise to a $80 \mathrm{bp}$ product. The lesion frequency of the mtDNA is calculated using the equation below ${ }^{90}$. Chloroquine (CQ) at $40 \mu \mathrm{M}$ was used as a positive control (Supplementary Table 5).

Lesion frequency per $16 \mathrm{~kb}=-\operatorname{Ln}\left(\frac{\text { Long PCR product/Short PCR product }}{\text { Mean of (Long PCR product/Short OCR product) from control }}\right)$

Transcriptional analysis of GEO datasets. A set of genes relevant to mitochondrial $\beta$-oxidation were collated, and then examined for differential gene expression (Supplementary Table 2). Published transcriptome data (NCBI GEO DataSets Accession numbers GSE53053; GSE69607; GSE84517; GSE46903; GSE35449; and GSE5099), regarding different macrophage phenotypes (M0 or M1 from both murine and human origin) generated using similar experimental conditions to those in the current study, were downloaded. All datasets were processed in $\mathrm{R}$ and using packages from Bioconductor. Affymetrix microarray data (GSE69607, GSE5099) were normalized and processed using Limma (3.40.6) and Oligo Bioconductor (1.48.0) packages ${ }^{91,92}$. Illumina beadchip microarray data (GSE46903, GSE35449) were normalized and processed using Limma and lumi (2.36.0) Bioconductor packages ${ }^{91,93}$. Paired-end reads from Illumina sequencing (GSE53053, GSE84517) were trimmed with Trim Galore (0.5.0 and assessed for quality using FastQC (0.11.8), using default parameters ${ }^{94,95}$. Reads were mapped to the reference sequence (human GRCh38 or mouse GRCm38) reference genome using STAR (2.7.0) and counts were assigned to transcripts using featureCounts (2.0.0) with the Ensembl gene build GTF (GRCh38.96 or GRCm38.84) ${ }^{96,97}$. Both the reference genome and GTF were downloaded from the Ensembl FTP site (http://www.ensembl.org/info/data/ftp/index.html/). Differential gene expression analyses used the DESeq2 (1.24.0) package ${ }^{98}$. Genes were discarded from the analysis differential expression if they failed to be significant (significance: adj.pval $<0.05$, Benjamini-Hochberg correction for multiple testing).

Analysis of human microarray data. Data from a published study were obtained, from GSE25504 ${ }^{57}$. Here, whole blood was analyzed using Illumina Human WholeGenome Expression BeadChip HT12v3 microarrays comprising 48,802 features (human gene probes) from neonates with proven microbiological evidence of infection from a usually sterile body site (35 controls, 26 bacterial confirmed sepsis cases). These patients also demonstrated significantly elevated expression markers that form part of the published classifier for sepsis ${ }^{57}$. Log2 expression data for the set of relevant genes (Supplementary Table 2) was compared, Student's $t$-test significance: adj.pval $<0.05$, Benjamini-Hochberg correction for multiple testing.

Analysis of mouse in vivo bacterial peritonitis gene expression. The SES model was carried out and lavage harvested at 3 or $6 \mathrm{~h}$ as described ${ }^{58}$, data are available as: E-MTAB-10739. Eight to twelve weeks old mixed-gender wild-type C57Bl/6 mice were from Charles River. Parietal peritoneal tissue was extracted at 3 and $6 \mathrm{~h}$ post SES administration, and collected using aseptic techniques. Two sections of lining were immediately snap-frozen in liquid $\mathrm{N}_{2}$ and stored at $-80^{\circ} \mathrm{C}$ prior to total RNA extraction. For this, peritoneal membrane sections $(80 \mathrm{mg})$ were dissociated in $1 \mathrm{ml}$ buffer RLT (QIAGEN) supplemented with $\beta$-mercaptoethanol (1:100 v:v) using a handheld electric homogenizer (Benchmark Scientific). Lysate was diluted 1:3 in distilled water and digested in $0.2 \mathrm{mg} / \mathrm{ml}$ proteinase- $\mathrm{K}$ (Invitrogen; 25530049) for $10 \mathrm{~min}$ at $55^{\circ} \mathrm{C}$. Lysate was cleared and RNA precipitated in $70 \%$ ethanol. Total RNA was extracted using the RNeasy minikit (QIAGEN; 24136) in accordance with the manufacturer's instructions. RNA was eluted in $50 \mu$ RNase-free water and quantified using a nanodrop 2000. The integrity and quality of RNA preparations was assessed using an Agilent 2100 bioanalyzer. Samples with an RNA integrity number exceeding 8 were used for library preparation (2-4 $\mu \mathrm{g}$ input). Cytoplasmic, mitochondrial, and ribosomal RNA was depleted using the Ribominus transcriptome isolation kit (Ambion; K155001). Libraries were prepared using the RNA-seq kit v2 (Life technologies; 4475936) and sequencing on an ion torrent (Thermo Fisher). Raw data were mapped using Torrent Suite ${ }^{\mathrm{TM}}$ (STAR and Bowie2 (2.3.4.3) aligners) using $\mathrm{mm} 10$ as the reference genome. Library quality was assessed using FastQC (0.11.8). Differential gene expression analysis was completed with DEseq2 (Bioconductor, 1.24.0). For the specific genes tested (Supplementary Table 2), Student's $t$-test was used, significance: adj.pval $<0.05$,

Benjamini-Hochberg correction for multiple testing. 
Analysis of 12-HETE incorporation into PLs by RAW264 cells. For MS precursor scanning, $1 \times 10^{6}$ RAW264 cells were seeded in a 6-well plate in DMEM supplemented with $10 \%$ FBS. The next day, cells were washed twice with PBS and $2 \mathrm{ml}$ serum-free RPMI (no phenol red) was added to each well. Cells were treated for $3 \mathrm{~h}$ with $100 \mathrm{ng} / \mathrm{ml}$ LPS, with and without $2.8 \mu \mathrm{g}$ 12(S)-HETE/ $10^{6}$ cells. Following 3-h incubation, cells were scraped into their media and immediately transferred to $2.5 \mathrm{ml}$ extraction solvent (hexane:isopropanol) and extracted as described in Methods. For subsequent experiments to quantify 12-HETE incorporated into PLs, $2 \times 10^{6}$ cells were seeded into 6-well plates in DMEM supplemented with $10 \%$ FBS. The next day, cells were washed twice with PBS and $1 \mathrm{ml}$ serum-free RPMI (no phenol red) was added to each well. Cells were treated for $3 \mathrm{~h}$ with and without $100 \mathrm{ng} / \mathrm{ml}$ LPS and with and without $1.4 \mu \mathrm{g}$ 12(S)-HETE/ $10^{6}$ cells. Following a 3-h incubation, cells were scraped directly into their culture media and immediately transferred to $2.5 \mathrm{ml}$ extraction solvent and extracted using the hexane:isopropanol method, as described in the Methods section. Lipids were analyzed on a Sciex 6500 QTrap, with chromatography as follows: Luna $3 \mu \mathrm{m} \mathrm{C18} 150 \times 2 \mathrm{~mm}$ column (Phenomenex, Torrance, CA) with a gradient of $50-100 \%$ B over $10 \mathrm{~min}$ followed by $30 \mathrm{~min}$ at $100 \% \mathrm{~B}$ (A, methanol:acetonitrile:water, $1 \mathrm{mM}$ ammonium acetate, 60:20:20; B, methanol, $1 \mathrm{mM}$ ammonium acetate) with a flow rate of $200 \mu \mathrm{l} / \mathrm{min}$. Source and MS conditions were as follows: CUR 35, IS-4500, TEM 500, GS1 40, GS2 30, DP-50, CE-38, CXP-11. First, a precursor scan (PREC 319.2) was performed in negative mode to determine a list of precursor $\mathrm{PE}$ ions present in the cell extract, scanning a mass range of $600-950 \mathrm{Da}$ at a scan rate of $1000 \mathrm{Da} / \mathrm{s}$. Following this, ten ions of interest were identified and EPI scans were performed for each precursor ion to obtain MS/MS spectra. Source and chromatography conditions were identical to the above. Product ions were scanned at $1000 \mathrm{Da} / \mathrm{s}$ with a mass range of $100-820 \mathrm{Da}$, using a dynamic fill time in the trap. Once candidate lipids were identified, extracts were analyzed in MRM mode, monitoring the precursor ion to the 12-HETE internal product ion $(\mathrm{m} / \mathrm{z}$ 179.1) (Supplementary Table 6). Samples were spiked with internal standards 15:0-18:1(d7) PE (10 ng) and 15:0-18:1(d7) PC (300 ng), and analytes quantified against a calibration curve using 18:0a/12-HETE-PE and 18:0a/12-HETE-PC as primary standards. MRMs were as follows: $m / z$ [M-H $]^{-}$738.6-179.1, 754.6-179.1, 764.6-179.1, 766.6-179.1, 780.6-179.1, 782.6-179.1, 808.7-179.1, 810.7-179.1 Chromatography and source conditions were the same as above. Peak inclusion criteria were those that had least 7 points across the peak and exceeded a signal:noise ratio of 5:1.

LC-MS/MS of free fatty acids (FFA). FFA were extracted from cell culture cells and supernatant $(1 \mathrm{ml})$ using the hexane:isopropanol method described above. An internal standard mix was added $(10 \mu \mathrm{l})$ to samples prior to extraction (Supplementary Table 7). FFA were derivatized in sample extracts according to Han et al. with modifications ${ }^{99}$. Dried extracts were reconstituted in methanol $(100 \mu \mathrm{l})$, then $50 \mu \mathrm{l} 3 \mathrm{NPH}(200 \mathrm{mM} 3-$ nitrophenyl-hydrazinein, 50/50 Methanol/ $\left.\mathrm{H}_{2} \mathrm{O}\right)$ and $50 \mu \mathrm{l}$ EDC/PYR (120 mM 1-Ethyl3-(3-dimethylaminopropyl)carbodiimide and $6 \%$ pyridine, $50 / 50 \mathrm{Methanol} / \mathrm{H}_{2} \mathrm{O}$ ) were added. Samples were vortexed then incubated for $30 \mathrm{~min}$ at $40{ }^{\circ} \mathrm{C}$. Excess derivatization reagents were quenched by the addition of $0.5 \%$ formic acid $(100 \mu \mathrm{L} ; 75 / 25 \mathrm{Methanol} /$ $\mathrm{H}_{2} \mathrm{O}$ ) and incubation at $40^{\circ} \mathrm{C}$ for $30 \mathrm{~min}$. Samples were aliquoted into HPLC vials for LC/MS/MS in MRM mode on a Nexera liquid chromatography system (Shimadzu) coupled to a QTRAP 4000 mass spectrometer with an ESI source (Sciex). Liquid chromatography was performed using a C18 reverse-phase column (Kinetex Polar C18 Column; $100 \AA, 100 \times 2.1 \mathrm{~mm}, 2.6 \mu \mathrm{m}$ particle size, Phenomenex). Two mobile phases were used: $100 \% \mathrm{H}_{2} \mathrm{O}+0.1 \%$ formic acid (mobile phase A) and $100 \%$ methanol + $0.1 \%$ formic acid (mobile phase B). The gradient was $15 \%$ B $0-2.47$ min, increasing to $55 \% \mathrm{~B}$ at $11.12 \mathrm{~min}$; then to $100 \% \mathrm{~B}$ at $20 \mathrm{~min}$; held at $100 \% \mathrm{~B}$ up to $32 \mathrm{~min}$; then $10 \mathrm{~min}$ re-equilibration at $15 \% \mathrm{~B}$. The flow rate was at $0.2 \mathrm{ml} / \mathrm{min}$ and injection volume $2 \mu \mathrm{l}$. Column temperature, $35^{\circ} \mathrm{C}$. MRM transitions, and MS settings (declustering potential and collision energy) for individual FFA are shown in Supplementary Table 7. FFA were detected in negative ion mode and the method was scheduled with fixed retention times and 40-s acquisition windows. Analytes were quantified based on the ratio of response to deuterated internal standards in comparison to externally generated standard curves for primary standards using MultiQuant software, V3.0.2.

Statistics. For lipidomics experiments comparing more than two conditions, one-way ANOVA with Tukey post hoc test is used (https://astatsa.com/OneWay_Anova_ with_TukeyHSD/_get_data/). Although all conditions are compared, our study is focused on the impact of CPT1 inhibition/silencing, so our text and figures generally show those comparisons. Where only two conditions are compared, Student's $t$-test was used. For the complete information on all comparisons, ANOVA and $t$-test results are also provided in our supplementary data file (Supplementary Data.xls). Where no significant difference was seen, no comparison bar is shown. Where lipids were not detected in some samples of a dataset, their values were replaced with corresponding 50\% LOQ values prior to conducting statistical analysis. These were calculated using the LOQ for the corresponding primary standards, taking into account sample volumes injected on the column. Figures show zero values, and all $50 \%$ LOQ values are provided (in red text) in supplementary data, where used, but the zero value was plotted on figures. If a lipid was not detected in any sample, it was not included in the dataset, and the full list of lipids measured in all samples is provided in Supplementary Data File 2. All $p$ values are provided in Source Data. For statistical analysis, Prizm (7-9) or Excel (2013, 2016, and office 365 versions) were used.
Reporting summary. Further information on research design is available in the Nature Research Reporting Summary linked to this article.

\section{Data availability}

The raw numbers for charts and graphs are available in the Source Data file whenever possible. All data are included in the article and its Supplementary Information, or are available from the authors upon reasonable requests. Source Data are provided with this paper.

Received: 4 August 2020; Accepted: 12 December 2021; Published online: 10 January 2022

\section{References}

1. Funk, C. D. Prostaglandins and leukotrienes: advances in eicosanoid biology. Science 294, 1871-1875 (2001).

2. Simmons, D. L., Botting, R. M. \& Hla, T. Cyclooxygenase isozymes: the biology of prostaglandin synthesis and inhibition. Pharm. Rev. 56, 387-437 (2004).

3. Smith, W. L., DeWitt, D. L. \& Garavito, R. M. Cyclooxygenases: structural, cellular, and molecular biology. Annu Rev. Biochem. 69, 145-182 (2000).

4. Serhan, C. N., Chiang, N. \& Van Dyke, T. E. Resolving inflammation: dual anti-inflammatory and pro-resolution lipid mediators. Nat. Rev. Immunol. 8, 349-361 (2008).

5. Capdevila, J. H., Falck, J. R. \& Imig, J. D. Roles of the cytochrome P450 arachidonic acid monooxygenases in the control of systemic blood pressure and experimental hypertension. Kidney Int. 72, 683-689 (2007).

6. Fleming, I. DiscrEET regulators of homeostasis: epoxyeicosatrienoic acids, cytochrome P450 epoxygenases and vascular inflammation. Trends Pharm. Sci. 28, 448-452 (2007).

7. Imig, J. D. Targeting epoxides for organ damage in hypertension. J. Cardiovasc. Pharm. 56, 329-335 (2010).

8. Roman, R. J. P-450 metabolites of arachidonic acid in the control of cardiovascular function. Physiol. Rev. 82, 131-185 (2002).

9. Spector, A. A. \& Norris, A. W. Action of epoxyeicosatrienoic acids on cellular function. Am. J. Physiol. Cell Physiol. 292, C996-C1012 (2007).

10. Williams, J. M. et al. 20-hydroxyeicosatetraeonic acid: a new target for the treatment of hypertension. J. Cardiovasc. Pharm. 56, 336-344 (2010).

11. Breyer, R. M. et al. Prostanoid receptors: subtypes and signaling. Annu. Rev. Pharm. Toxicol. 41, 661-690. (2001).

12. Cheng, H. F. \& Harris, R. C. Cyclooxygenases, the kidney, and hypertension. Hypertension 43, 525-530 (2004).

13. Hao, C. M. \& Breyer, M. D. Physiological regulation of prostaglandins in the kidney. Annu. Rev. Physiol. 70, 357-377. (2008).

14. Capra, V. et al. Cysteinyl-leukotrienes and their receptors in asthma and other inflammatory diseases: critical update and emerging trends. Med. Res. Rev. 27, 469-527 (2007).

15. Ribeiro, J. D., Toro, A. A. \& Baracat, E. C. Antileukotrienes in the treatment of asthma and allergic rhinitis. J. Pediatr. (Rio J.) 82, S213-S221 (2006).

16. Sharma, J. N. \& Mohammed, L. A. The role of leukotrienes in the pathophysiology of inflammatory disorders: is there a case for revisiting leukotrienes as therapeutic targets? Inflammopharmacology 14, 10-16 (2006).

17. Diczfalusy, U. et al. Metabolism of prostaglandin F2 alpha in Zellweger syndrome. Peroxisomal beta-oxidation is a major importance for in vivo degradation of prostaglandins in humans. J. Clin. Invest. 88, 978-984 (1991).

18. Wigren, J. et al. Metabolism of 12(S)-hydroxy-5,8,10,14-eicosatetraenoic acid by kidney and liver peroxisomes. J. Lipid Res. 34, 625-631 (1993).

19. Diczfalusy, U. Beta-oxidation of eicosanoids. Prog. Lipid Res. 33, 403-428. (1994).

20. Spector, A. A., Gordon, J. A. \& Moore, S. A. Hydroxyeicosatetraenoic acids (HETEs). Prog. Lipid Res. 27, 271-323 (1988).

21. Benedetto, C., McDonald-Gibson, R. G., Nigam, S., \& Slater, T. F. Prostaglandins and Related Substances: A Practical Approach. 1988 (Oxford, Washington: IRL Press, 1987).

22. Eaton, S., Bartlett, K. \& Pourfarzam, M. Mammalian mitochondrial betaoxidation. Biochem. J. 320, 345-357 (1996).

23. Wang, H. Y. \& Schulz, H. Beta-oxidation of polyunsaturated fatty acids with conjugated double bonds. Mitochondrial metabolism of octa-2,4,6-trienoic acid. Biochem. J. 264, 47-52 (1989).

24. Houten, S. M. et al. The biochemistry and physiology of mitochondrial fatty acid beta-oxidation and its genetic disorders. Annu. Rev. Physiol. 78, 23-44 (2016).

25. Kaminski, M. M. et al. Polarization and asymmetry in T cell metabolism. Semin. Immunol. 28, 525-534 (2016). 
26. O'Neill, L. A. \& Pearce, E. J. Immunometabolism governs dendritic cell and macrophage function. J. Exp. Med. 213, 15-23 (2016).

27. Slatter, D. A. et al. Mapping the human platelet lipidome reveals cytosolic phospholipase A2 as a regulator of mitochondrial bioenergetics during activation. Cell Metab. 23, 930-944 (2016).

28. Palmieri, E. M. et al. Nitric oxide orchestrates metabolic rewiring in M1 macrophages by targeting aconitase 2 and pyruvate dehydrogenase. Nat. Commun. 11, 698 (2020).

29. O'Neill, L. A., Kishton, R. J. \& Rathmell, J. A guide to immunometabolism for immunologists. Nat. Rev. Immunol. 16, 553-565 (2016).

30. Thapa, B. \& Lee, K. Metabolic influence on macrophage polarization and pathogenesis. BMB Rep. 52, 360-372 (2019).

31. Dioszeghy, V. et al. 12/15-Lipoxygenase regulates the inflammatory response to bacterial products in vivo. J. Immunol. 181, 6514-6524 (2008).

32. Cornicelli, J. A. et al. Mouse peritoneal macrophages contain abundant omega-6 lipoxygenase activity that is independent of interleukin-4. Arterioscler. Thromb. Vasc. Biol. 16, 1488-1494 (1996).

33. Murray, P. J. et al. Macrophage activation and polarization: nomenclature and experimental guidelines. Immunity 41, 14-20 (2014).

34. Kriska, T. et al. Deactivation of 12(S)-HETE through (omega-1)hydroxylation and beta-oxidation in alternatively activated macrophages. $J$. Lipid Res. 59, 615-624 (2018).

35. Pawlowski, N. A. et al. Uptake and metabolism of monohydroxyeicosatetraenoic acids by macrophages. J. Exp. Med. 155, 1653-1664 (1982)

36. Divakaruni, A. S. et al. Etomoxir inhibits macrophage polarization by disrupting CoA homeostasis. Cell Metab. 28, 490-503.e7 (2018).

37. Raud, B. et al. Etomoxir actions on regulatory and memory $\mathrm{T}$ cells are independent of Cptla-mediated fatty acid oxidation. Cell Metab. 28, 504-515.e7 (2018).

38. Yao, C.-H. et al. Identifying off-target effects of etomoxir reveals that carnitine palmitoyltransferase I is essential for cancer cell proliferation independent of B-oxidation. PLoS Biol. 16, e2003782 (2018).

39. Zhang, J., Frerman, F. E. \& Kim, J. J. Structure of electron transfer flavoprotein-ubiquinone oxidoreductase and electron transfer to the mitochondrial ubiquinone pool. Proc. Natl Acad. Sci. USA. 103, 16212-16217 (2006).

40. Jha, A. K. et al. Network integration of parallel metabolic and transcriptional data reveals metabolic modules that regulate macrophage polarization. Immunity 42, 419-430 (2015).

41. Ryan, D. G. \& O’Neill, L. A. J. Krebs cycle rewired for macrophage and dendritic cell effector functions. FEBS Lett. 591, 2992-3006 (2017).

42. Qian, X., Zhang, J. \& Liu, J. Tumor-secreted PGE2 inhibits CCL5 production in activated macrophages through cAMP/PKA signaling pathway. J. Biol. Chem. 286, 2111-2120 (2011).

43. Sheppe, A. E. F. et al. PGE2 augments inflammasome activation and M1 polarization in macrophages infected with Salmonella Typhimurium and Yersinia enterocolitica. Front Microbiol. 9, 2447 (2018).

44. Strassheim, D. et al. Prostacyclin inhibits IFN-gamma-stimulated cytokine expression by reduced recruitment of CBP/p300 to STAT1 in a SOCS-1independent manner. J. Immunol. 183, 6981-6988 (2009).

45. Takayama, K. et al. Prostaglandin E2 suppresses chemokine production in human macrophages through the EP4 receptor. J. Biol. Chem. 277, 44147-44154 (2002).

46. Heming, M. et al. Peroxisome proliferator-activated receptor- $\gamma$ modulates the response of macrophages to lipopolysaccharide and glucocorticoids. Front. Immunol. 9, 893 (2018).

47. Loynes, C. A. et al. PGE2 production at sites of tissue injury promotes an antiinflammatory neutrophil phenotype and determines the outcome of inflammation resolution in vivo. Sci. Adv. 4, eaar8320 (2018).

48. Pitts, M. G. \& D'Orazio, S. E. F. Prostaglandin E2 inhibits the ability of neutrophils to kill Listeria monocytogenes. J. Immunol. 202, 3474-3482 (2019).

49. Avila-Roman, J. et al. Microalgae-derived oxylipins decrease inflammatory mediators by regulating the subcellular location of NFkappaB and PPARgamma. Pharm. Res 128, 220-230 (2018).

50. Dunn, S. E. et al. Correction: peroxisome proliferator-activated receptor (PPAR)alpha expression in $\mathrm{T}$ cells mediates gender differences in development of T cell-mediated autoimmunity. J. Exp. Med. 215, 1765 (2018).

51. Kronke, G. et al. 12/15-Lipoxygenase counteracts inflammation and tissue damage in arthritis. J. Immunol. 183, 3383-3389 (2009).

52. Kumar, N. et al. 15-Lipoxygenase metabolites of alpha-linolenic acid, [13-(S)HPOTrE and 13-(S)-HOTrE], mediate anti-inflammatory effects by inactivating NLRP3 inflammasome. Sci. Rep. 6, 31649 (2016).

53. Sun, L. et al. 12/15-Lipoxygenase metabolites of arachidonic acid activate PPARgamma: a possible neuroprotective effect in ischemic brain. J. Lipid Res. 56, 502-514. (2015).
54. Wendell, S. G. et al. 15-Hydroxyprostaglandin dehydrogenase generation of electrophilic lipid signaling mediators from hydroxy omega-3 fatty acids. $J$. Biol. Chem. 290, 5868-5880 (2015).

55. Yamada, H. et al. Hydroxyeicosapentaenoic acids from the Pacific krill show high ligand activities for PPARs. J. Lipid Res. 55, 895-904 (2014).

56. Hurst, S. M. et al. Il-6 and its soluble receptor orchestrate a temporal switch in the pattern of leukocyte recruitment seen during acute inflammation. Immunity 14, 705-714 (2001).

57. Smith, C. L. et al. Identification of a human neonatal immune-metabolic network associated with bacterial infection. Nat. Commun. 5, 4649 (2014).

58. Fielding, C. A. et al. Interleukin- 6 signaling drives fibrosis in unresolved inflammation. Immunity 40, 40-50 (2014).

59. Beyer, M. et al. High-resolution transcriptome of human macrophages. PLoS One 7, e45466 (2012)

60. Huang, S. C. et al. Cell-intrinsic lysosomal lipolysis is essential for alternative activation of macrophages. Nat. Immunol. 15, 846-855 (2014).

61. Jablonski, K. A. et al. Novel markers to delineate murine M1 and M2 macrophages. PLoS One 10, e0145342 (2015).

62. Martinez, F. O. et al. Transcriptional profiling of the human monocyte-tomacrophage differentiation and polarization: new molecules and patterns of gene expression. J. Immunol. 177, 7303-7311 (2006).

63. Piccolo, V. et al. Opposing macrophage polarization programs show extensive epigenomic and transcriptional cross-talk. Nat. Immunol. 18, 530-540 (2017).

64. Xue, J. et al. Transcriptome-based network analysis reveals a spectrum model of human macrophage activation. Immunity 40, 274-88. (2014).

65. Mathur, S. N., Albright, E. \& Field, F. J. 12-Hydroxyeicosatetraenoic acid is metabolized by beta-oxidation in mouse peritoneal macrophages. Identification of products and proposed pathway. J. Biol. Chem. 265, 21048-21055 (1990).

66. Lu, R. et al. Cloning, in vitro expression, and tissue distribution of a human prostaglandin transporter cDNA(hPGT). J. Clin. Invest. 98, 1142-1149 (1996).

67. Bennett, M. J. \& Santani, A. B. Carnitine palmitoyltransferase 1A deficiency. In GeneReviews((R)) (eds Adam, M. P. et al.) https://www.ncbi.nlm.nih.gov/ books/NBK1527/ (Seattle (WA), 1993-2021).

68. Gessner, B. D. et al. Evidence for an association between infant mortality and a carnitine palmitoyltransferase 1A genetic variant. Pediatrics 126, 945-951 (2010).

69. Sinclair, G. et al. The p.P479L variant in CPT1A is associated with infectious disease in a BC First Nation. Paediatr. Child Health 24, el11-e115 (2019).

70. Ellis, J. M. et al. Adipose acyl-CoA synthetase-1 directs fatty acids toward beta-oxidation and is required for cold thermogenesis. Cell Metab. 12, 53-64 (2010).

71. Ellis, J. M. et al. Mouse cardiac acyl coenzyme a synthetase 1 deficiency impairs fatty acid oxidation and induces cardiac hypertrophy. Mol. Cell Biol. 31, 1252-1262 (2011).

72. Grevengoed, T. J. et al. Loss of long-chain acyl-CoA synthetase isoform 1 impairs cardiac autophagy and mitochondrial structure through mechanistic target of rapamycin complex 1 activation. FASEB J. 29, 4641-4653 (2015).

73. Grevengoed, T. J. et al. Acyl-CoA synthetase 1 deficiency alters cardiolipin species and impairs mitochondrial function. J. Lipid Res. 56, 1572-1582 (2015).

74. Lee, K., Kerner, J. \& Hoppel, C. L. Mitochondrial carnitine palmitoyltransferase 1a (CPT1a) is part of an outer membrane fatty acid transfer complex. J. Biol. Chem. 286, 25655-25662 (2011).

75. Li, L. O. et al. Compartmentalized acyl-CoA metabolism in skeletal muscle regulates systemic glucose homeostasis. Diabetes 64, 23-35 (2015).

76. Young, P. A. et al. Long-chain acyl-CoA synthetase 1 interacts with key proteins that activate and direct fatty acids into niche hepatic pathways. J. Biol. Chem. 293, 16724-16740 (2018).

77. Klett, E. L. et al. Long-chain acyl-CoA synthetase isoforms differ in preferences for eicosanoid species and long-chain fatty acids. J. Lipid Res. 58, 884-894 (2017)

78. Roelands, J. et al. Long-chain Acyl-CoA synthetase 1 role in sepsis and immunity: perspectives from a parallel review of public transcriptome datasets and of the literature. Front. Immunol. 10, 2410 (2019).

79. Janssen, U. et al. Human mitochondrial 3,2-trans-enoyl-CoA isomerase (DCI): gene structure and localization to chromosome 16p13.3. Genomics $\mathbf{2 3}$, 223-228 (1994).

80. Rasmussen, A. L. et al. Systems virology identifies a mitochondrial fatty acid oxidation enzyme, dodecenoyl coenzyme A delta isomerase, required for hepatitis C virus replication and likely pathogenesis. J. Virol. 85, 11646-11654 (2011).

81. Nomura, M. et al. Fatty acid oxidation in macrophage polarization. Nat. Immunol. 17, 216-217 (2016).

82. Wanders, R. J. A., Waterham, H. R. \& Ferdinandusse, S. Metabolic interplay between peroxisomes and other subcellular organelles including mitochondria and the endoplasmic reticulum. Front. Cell Developmental Biol. 3, 83 (2016). 
83. Gordon, J. A., Figard, P. H. \& Spector, A. A. Identification of the major metabolite of 12-HETE produced by renal tubular epithelial cells. J. Lipid Res. 30, 731-738 (1989).

84. Gordon, J. A., Figard, P. H. \& Spector, A. A. Hydroxyeicosatetraenoic acid metabolism in cultured human skin fibroblasts. Evidence for peroxisomal beta-oxidation. J. Clin. Invest. 85, 1173-1181 (1990).

85. Kitamura, T. et al. Retrovirus-mediated gene transfer and expression cloning: powerful tools in functional genomics. Exp. Hematol. 31, 1007-1014 (2003).

86. Ipseiz, $\mathrm{N}$. et al. Effective in vivo gene modification in mouse tissue-resident peritoneal macrophages by intraperitoneal delivery of lentiviral vectors. Mol. Ther. Methods Clin. Dev. 16, 21-31 (2020).

87. Ostermann, A. I., Willenberg, I. \& Schebb, N. H. Comparison of sample preparation methods for the quantitative analysis of eicosanoids and other oxylipins in plasma by means of LC-MS/MS. Anal. Bioanal. Chem. 407, 1403-1414 (2015).

88. Kolde, R. Pheatmap: pretty heatmaps 2012 .

89. Davies, L. C. et al. Peritoneal tissue-resident macrophages are metabolically poised to engage microbes using tissue-niche fuels. Nat. Commun. 8, 2074 (2017).

90. Redmann, M. et al. Methods for assessing mitochondrial quality control mechanisms and cellular consequences in cell culture. Redox Biol. 17, 59-69 (2018).

91. Ritchie, M. E. et al. limma powers differential expression analyses for RNAsequencing and microarray studies. Nucleic Acids Res. 43, e47 (2015).

92. Carvalho, B. S. \& Irizarry, R. A. A framework for oligonucleotide microarray preprocessing. Bioinformatics 26, 2363-2367 (2010).

93. Du, P., Kibbe, W. A. \& Lin, S. M. lumi: a pipeline for processing Illumina microarray. Bioinformatics 24, 1547-1548 (2008).

94. Kreuger, F. Trim Galore. https://www.bioinformatics.babraham.ac.uk/ projects/trim_galore/ (2019).

95. Andrews, S. FastQC. https://www.bioinformatics.babraham.ac.uk/projects/ fastqc/ (2019).

96. Dobin, A. et al. STAR: ultrafast universal RNA-seq aligner. Bioinformatics 29, 15-21 (2012).

97. Liao, Y., Smyth, G. K. \& Shi, W. featureCounts: an efficient general purpose program for assigning sequence reads to genomic features. Bioinformatics 30, 923-930 (2013).

98. Love, M. I., Huber, W. \& Anders, S. Moderated estimation of fold change and dispersion for RNA-seq data with DESeq2. Genome Biol. 15, 550 (2014).

99. Han, J. et al. An isotope-labeled chemical derivatization method for the quantitation of short-chain fatty acids in human feces by liquid chromatographytandem mass spectrometry. Anal. Chim. Acta 854, 86-94 (2015).

\section{Acknowledgements}

Funding from Wellcome Trust (094143/Z/10/Z) and European Research Foundation (LipidArrays) is gratefully acknowledged (V. B. O.). V. B. O. is a Royal Society Wolfson Research Merit Award Holder and acknowledges funding for LIPID MAPS from Wellcome Trust (203014/Z/16/Z). Ser Cymru Project Sepsis grant funded by WG/EUERDF (P. G., V. B. O.). P. R. T. is funded by a Wellcome Trust Investigator Award $(107964 / Z / 15 / Z)$ and the UK Dementia Research Institute. M. A. C. is funded by BBSRC Discovery Fellowship (BB/T009543/1). V.D.-U. acknowledges The Nathan Shock Center P30 AG05088. Kidney Research UK (RP-024-20160304, S.A.J.), Versus Arthritis
(Reference 20770 awarded to S.A.J., V.O.D.). M.A.C. is supported by BBSRC Discovery Fellowship (BB/T009543/1). We gratefully acknowledge expert input from Alan Brash, Vanderbilt University, relating to lipid structural elucidation, James Burston for supporting analysis of mitochondrial function, Widad Dantoft for provision of the human sepsis dataset, William Watkins for assistance with statistical analysis, and our colleague Javier Uceda Fernandez (deceased), Cardiff University for the murine transcriptomics dataset.

\section{Author contributions}

M.M., K.K., L.C.D., V.J.T., P.R.S.R., G.A.B., C.H., P.K., M.R., J.E.M.L., M.A.C., M.A., M.G., and D.A.W. conducted experiments; M.M., K.K., C.H., L.C.D., J.E. M.L., P.G., V.D.-U., D.A.W., S.A.J., and P.R.T. designed experiments; R.A., S.D., R.C.M., M.M., K.K. V.J.T., P.R.S.R., G.A.B., C.H., P.K., M.R., D.A.W., J.M.L., and L.C.D. analyzed data; N.H.S. and S.M. implemented and supported data analysis; V.O.D. drafted the manuscript and all authors edited the manuscript.

\section{Competing interests}

P.K. is an employee of Cayman Chemical. All other authors declare no competing interests.

\section{Additional information}

Supplementary information The online version contains supplementary material available at https://doi.org/10.1038/s41467-021-27766-8.

Correspondence and requests for materials should be addressed to Daniel A. White or Valerie B. O’Donnell.

Peer review information Nature Communications thanks the anonymous, reviewer(s) for their contribution to the peer review of this work. Peer reviewer reports are available.

Reprints and permission information is available at http://www.nature.com/reprints

Publisher's note Springer Nature remains neutral with regard to jurisdictional claims in published maps and institutional affiliations.

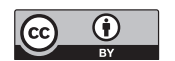

Open Access This article is licensed under a Creative Commons Attribution 4.0 International License, which permits use, sharing, adaptation, distribution and reproduction in any medium or format, as long as you give appropriate credit to the original author(s) and the source, provide a link to the Creative Commons license, and indicate if changes were made. The images or other third party material in this article are included in the article's Creative Commons license, unless indicated otherwise in a credit line to the material. If material is not included in the article's Creative Commons license and your intended use is not permitted by statutory regulation or exceeds the permitted use, you will need to obtain permission directly from the copyright holder. To view a copy of this license, visit http://creativecommons.org/ licenses/by/4.0/.

(C) The Author(s) 2022 